\title{
DIMENSIONES DE LA CALIDAD DEL SERVICIO DE LOS COMEDORES DE LA UNIVERSIDAD DE COSTA RICA DESDE LA EXPERIENCIA DE LAS PERSONAS USUARIAS
}

\section{DIMENSIONS OF SERVICE QUALITY OF THE DINING SERVICES OF THE UNI- VERSITY OF COSTA RICA FROM THE EXPERIENCE OF THE USERS}

\author{
Laura Milena Andrade-Pérez ${ }^{1}$ \\ Johnny Cartín-Quesada ${ }^{2}$ \\ M. Gabriela Montero-Rodríguez ${ }^{3}$ \\ Ana Beatriz Avendaño-Castro ${ }^{4}$
}

Recibido: $16 / 11 / 2020$

Aprobado: 16/12/2021

\section{RESUMEN}

La calidad que brindan los servicios de alimentación (SA) dentro del campus universitario juega un papel determinante en el estilo de vida y hábitos alimentarios de la población estudiantil. Objetivo: Analizar las dimensiones de la calidad de servicio de los SA de la Universidad Costa Rica, según la experiencia de las personas usuarias. Metodología: Involucra un estudio descriptivo, transversal con el instrumento adaptado del DINESERV a 409 usuarios del campus para evaluar el servicio recibido. Se realizó análisis descriptivo y análisis inferencial para determinar diferencias para cada dimensión por edad y ocupación así como pruebas de Kruskal-Wallis y pruebas post hoc. Se determinó la contribución de las dimensiones a la variabilidad de la experiencia de la calidad de servicio usando un modelo de regresión lineal múltiple. En todos los casos se aceptó un 95\% de confianza. Resultados: Las dimensiones de confiabilidad y garantía fueron las mejores calificadas y empatía la que obtuvo menor puntaje. La edad del usuario y la ocupación no afectaron significativamente la percepción de la calidad del servicio. Conclusiones: La calidad del servicio desde la perspectiva todas las personas usuarias informa la importancia de enfatizar en los procesos de contratación a terceros los aspectos relacionados a la dimensión tangible, seguido de garantía, confiabilidad y empatía.

PALABRAS CLAVE: DINESERV, DIMENSIONES DE LA CALIDAD, CALIDAD DEL SERVICIO, EXPERIENCIA DEL USUARIO, COMEDOR UNIVERSITARIO

CLASIFICACIÓN JEL: I0, M110, M310, Z310

1 Universidad de Costa Rica, Escuela de Nutrición; Código Postal: 11501-2060; San José, Costa Rica; laura.andrade@ucr.ac.cr

2 Universidad Estatal a Distancia, Programa de investigación para los fundamentos de la educación superior (PROIFED)/Vicerrectoría de Investigación; Código postal 474-2050; San José, Costa Rica; jcartinq@uned.ac.cr

3 Universidad de Costa Rica, Escuela de Nutrición; Código Postal: 11501-2060; San José, Costa Rica; maría.monterorodriguez@ucr.ac.cr

4 Universidad de Costa Rica, Escuela de Nutrición; Código Postal: 11501-2060; San José, Costa Rica; ana.avendano@ucr.ac.cr 


\begin{abstract}
The quality of food services (SA) within an university campus plays a determining role in the lifestyle and eating habits of the student population. Objective: To analyze the dimensions of the quality of service of the SA of the Universidad Costa Rica, according to the experience of the users. Methodology: It involves a descriptive, cross-sectional study with the adapted DINESERV instrument to 409 campus users to evaluate the service received. Descriptive analysis and inferential analysis were performed to determine differences for each dimension by age and occupation, as well as Kruskal-Wallis tests and post hoc tests. The contribution of the dimensions to the variability of the service quality experience was determined using multiple linear regression model. In all cases, 95\% confidence was accepted. Results: The reliability and guarantee dimensions were the best rated and empathy the one with the lowest score. The user's age and occupation did not significantly affect the perception of the quality of the service. Conclusions: The quality of the service from the perspective of all users informs the importance of emphasizing in the contracting processes to third parties the aspects related to the tangible dimension, followed by guarantee, reliability and empathy.
\end{abstract}

KEYWORDS: DINESERV, DIMENSIONS OF QUALITY, QUALITY OF SERVICE, USER EXPERIENCE, UNIVERSITY DINNING.

JEL CLASSIFICATION: I0, M110, M310, Z310

\title{
I. INTRODUCCIÓN
}

Los servicios de alimentación (SA) universitarios juegan un papel importante en la vida diaria de gran cantidad de estudiantes en especial por largas permanencias en el campus, y en ocasiones provienen de lugares lejanos o no disponen de tiempo suficiente para comer o preparar alimentos saludables (Espinoza et al., 2011) y por tanto deben hacer uso de los comedores que existen en el campus de las instituciones educativas.

Específicamente, la evaluación de la calidad en los SA, ha tomado importancia a través de los años conforme la economía se ha ido transformando en una economía orientada al servicio, donde estos establecimientos, globalmente, representan el 20\% del ingreso del sector de servicios, $y$ poseen una tasa de crecimiento anual de 3\% (Tzeng \& Chang, 2011).

En la Universidad de Costa Rica (UCR), existen comedores y cafeterías, donde se busca asegurar el acceso a una alimentación de calidad e inocua, y además, favorecer la promoción de la salud. Los SA en la Sede Rodrigo Facio cubren las necesidades de alimentación de aproximadamente 5.000 personas cada día, correspondientes a estudiantes, profesores, funcionarios y visitantes (García Bravo \& López Sedó, 2015).

El presente estudio, resulta innovador puesto que es la primera vez que se evalúan estos servicios para conocer la opinión de las personas usuarias, de forma que contribuya a la toma de decisiones que orienten la gestión óptima de los servicios ofrecidos por parte de las instancias responsables. El propósito del mismo es poder identificar cuales son las dimensiones que deben ser priorizadas en la toma de decisiones por las autoridades universitarias de forma que se garantice la calidad del servicio en los SA y contribuya a la priorización de los criterios para la contratación y auditorías de los concesionario.

\section{A. Servicio de Alimentación Institucional en el ámbito universitario}

Los servicios de alimentación institucional son aquellos que preparan y sirven comidas, como un servicio de apoyo a las actividades regulares que desarrollan las personas en instituciones como empresas, instituciones educativas, centros de cuido, y hospitales entre otros. Existen autores que 
afirman que más allá de ser una fuente de alimentos, puesto que los SA son un elemento esencial que afecta la calidad de vida universitaria, puesto que la alimentación, además de contribuir al bienestar físico y ser una fuente de placer, tiene relación cultural y social; lo que ha promovido la revisión de sus operaciones para determinar si estos responden a las necesidades básicas de sus usuarios (Kwun, 2011).

El servicio de alimentación ofrecido por la UCR es un servicio contratado a terceros el cual se lleva a cabo mediante licitaciones públicas por medio de la oficina de Servicios Generales (Oficina de Servicios Generales, 2012). Estas se subdividen en apartados constituidos por cláusulas de acatamiento obligatorio, entre los cuales se encuentran: las condiciones de uso de las instalaciones y activos, disposiciones en cuanto a los trabajadores contratados por el adjudicatario, las obligaciones del contratista, las disposiciones sanitarias y manipulación de alimentos (Oficina de Suministros de la Universidad de Costa Rica, 2019; Ramírez, 2014).

Como parte de las especificaciones contenidas en los carteles de licitación, se encuentran aspectos relacionados con la oferta de los SA universitarios, la cual está determinada principalmente por disposiciones nutricionales en las licitaciones de prestación de servicios (Universidad de Costa Rica, 2019), no obstante se carece en las especificaciones acerca de dimensiones de la calidad del servicio, lo cual justifica la presente investigación.

\section{B. Dimensiones de la calidad de servicio}

Con la calidad de servicio brindada en un SA, se pretende alcanzar una experiencia global satisfactoria para el cliente, tomando en consideración que según Barrios (2012), el término experiencia se utiliza como el registro a nivel de cuerpo y mente del afecto, el pensamiento o la emoción que se percibe a través de los sentidos, cuando se participa en una actividad.

Un estudio realizado en la Universidad Carabobo en Venezuela, ha podido evidenciar mediante entrevistas a las personas usuarias de su comedor estudiantil, que hay ciertos aspectos referentes a la calidad del servicio que afectan la satisfacción de estos. Para conocer la opinión de las personas usuarias con respecto a la calidad de servicio y satisfacción del cliente, en el estudio usaron un instrumento basado en el modelo SERVQUAL para medir las percepciones de la calidad de los servicios universitarios, incluyéndose además una escala basado en el American Customer Satisfaction Index (ACSI) para medir la satisfacción de las personas usuarias (Mejías et al., 2012).

El modelo cuantitativo multidimensional denominado DINESERV, es una herramienta desarrollada por Stevens et al. (1995), derivado de la metodología SERVQUAL o Service Quality permite evaluar la calidad de servicio de manera general o por cada una de sus dimensiones, permitiendo así identificar cuáles aspectos requieren de mayor atención. Conocer la opinión de sus usuarios sobre los productos y servicio que reciben, resulta fundamental para la toma de decisiones, puesto que al satisfacer sus necesidades $y$ expectativas, es posible construir opiniones positivas (Palacios Palacios, 2014).

Esta herramienta consiste en un instrumento que reúne una serie de preguntas que tienen como objetivo evaluar la calidad de servicio vista desde cinco dimensiones. Las cinco dimensiones que incluye la evaluación son las siguientes (Lee \& Hing, 1995):

- Tangibles: Son aspectos físicos que el usuario percibe de la organización, tales como limpieza y modernidad, los cuales son evaluadas en los elementos, personas, infraestructura y objetos. Se incluye instalaciones físicas, equipos y la apariencia del personal del establecimiento.

- Confiabilidad: Es la capacidad de las organizaciones para realizar el servicio prometido con precisión, considerando aspectos específicos de los alimentos ordenados por el usuario. 
- Capacidad de respuesta: Es la disposición de los proveedores de servicios para ayudar a las personas usuarias y ofrecer un servicio rápido.

- Garantía: Se considera como el conocimiento y cortesía de los empleados y su capacidad para inspirar confianza y seguridad.

- Empatía: Incluye la simpatía y atención individualizada que la empresa ofrece a sus usuarios.

Estas cinco dimensiones de calidad se califican, según la percepción de las personas usuarias de los SA, para otorgar una calificación de calidad de servicio. La premisa que plantean los creadores de la herramienta es que, a mayor calificación, mayor será la probabilidad de que las personas usuarias regresen al SA y de que el establecimiento sea recomendado a otras personas, lo que en última instancia se traduce en una mayor ganancia para el concesionario y retorno de la inversión para las universidades (Stevens et al., 1995).

Para los propósitos de este estudio, se utilizó el instrumento DINESERV adaptado y traducido por Aguirre, J., y Rodríguez, M. en 2011; el cual mediante el análisis de confiabilidad Alpha de Cronbach's 0,9352 ; asimismo todos los componentes individuales y sus variables reportaron niveles satisfactorios y superiores a 0,90 Alpha de Cronbach's, cifra que se considera de confiabilidad adecuada. Lo anterior brinda la certeza de que los resultados con la versión en español son igual de consistentes con los obtenidos por la versión en inglés; $y$ denota que el instrumento utilizado responde a las condiciones nacionales para valorar la satisfacción de la calidad del servicio (Aguirre, \& Rodríguez, 2011).

\section{METODOLOGÍA}

Este estudio se considera de tipo descriptivo, transversal, y es una investigación exploratoria, puesto que el tema de dimensiones de la calidad en los servicios de alimentación institucional ha sido poco estudiado en el ámbito nacional.

\section{Población y muestra}

Estuvo constituida 5000 usuarios de todos los servicios de alimentación de la universidad (Universidad de Costa Rica, 2019). A partir de esta población se seleccionó una muestra estratificada, proporcional y aleatoria de 409 usuarios que usaron el servicio de comedor en el tiempo de comida del almuerzo en 2016 en ocho comedores que se encuentran en la Sede Rodrigo Facio. Se estimó por medio de una fórmula estadística para poblaciones finitas con un nivel de confianza de $95 \%$ y error de muestreo de 5\%. Se eligió el tiempo de comida del almuerzo puesto que es el momento de mayor afluencia de usuarios, según datos proporcionados por la Sección de Servicios Contratados de la Oficina de Servicios Generales de la Universidad de Costa Rica.

\section{Técnicas de recolección}

Se recolectaron los datos usando un instrumento conformado por un cuestionario autoadministrado a estudiantes, funcionarios y público en general que se encontraba presente en los diferentes SA de la UCR en el momento del estudio. El instrumento constituía de dos partes: el perfil del usuario el cual incluyó edad, relación con la universidad, si estudia y/o trabaja y frecuencia de asistencia al comedor. Luego se incluyeron las preguntas que evaluaron la experiencia de estos al utilizar dichos servicios. Dicho cuestionario corresponde al instrumento adaptado de la traducción de Aguirre y Rodríguez (2011), que incluye la evaluación de las dimensiones de calidad de 
servicio con preguntas cerradas: dimensión tangibles: de la pregunta 1 - 10, confiabilidad: 11 - 15 , capacidad de respuesta: 16 - 18 garantía: 19 - 24 empatía: 25 - 29. Cada ítem fue valorado mediante escalas de Likert, de menor a mayor grado de satisfacción, siendo 1 "Muy en desacuerdo" y 10 "Completamente de acuerdo". Además se incluyó en la pregunta 29 realizar una valoración general de la calidad del servicio, siendo una escala de 1 "Deficiente" a 10 "Excelente". Para una mejor comprensión del la pregunta y se excluyó lo relacionado a estacionamiento, baños y meseros, puesto que en este entorno no eran pertinentes consultarlas y se le realizaron ajustes de redacción a las preguntas (Anexo 1).

Se recolectó la información durante el tiempo de comida del almuerzo, los días más concurridos: martes, miércoles y jueves. La persona encargada de entregar los cuestionarios, realizaba una secuencia aleatoria en la que al azar elegía un número y ese sería el factor que contaba entre los asistentes al lugar para abordar a posibles participantes, quienes de aceptar voluntariamente se les solicitaba el consentimiento y luego se les distribuía el cuestionario a las personas usuarias para ser completado durante su estancia en el comedor.

\section{Procesamiento de análisis}

Los datos recolectados se analizaron mediante estadística descriptiva. Los mismos fueron tabulados y analizados por medio del software Microsoft ${ }^{\circledR}$ Excel ${ }^{\circledR}$ para Microsoft 365, a los puntajes para cada dimensión, se les calculó promedio y desviación estándar para gráficos y cuadros para las dimensiones de la calidad de acuerdo con la edad y ocupación del usuario. Para el análisis inferencial se realizó el programa programa RStudio 2021.09 para determinar las diferencias entre las dimensiones por edad y ocupación, para ello se realizaron pruebas de validación de supuestos posterior a esto se aplicó pruebas de Kruskal-Wallis dado el comportamiento de los datos para comparar las dimensiones de servicio y post hoc pruebas de Nemenyi. Para todo de aceptó un error de 5\%. Finalmente se realizó un modelo de regresión lineal múltiple para medir la contribución de las dimensiones a la variabilidad de la experiencia de la calidad de servicio.

\section{RESULTADOS}

El concepto de calidad puede ser visto de dos maneras, primero como las características de un producto o servicio que le confieren su habilidad de satisfacer necesidades específicas o implícitas y también, como un producto o servicio que se encuentra libre de defectos. Adicionalmente, la calidad de servicio se ha definido como la manera en que se cumple con las expectativas de servicio de los clientes, es por esta misma razón que el concepto se ha considerado relativo (Kim et al., 2010). Asimismo, Vera y Trujillo León en 2017 mencionan que varios autores indican que "la calidad en el servicio es un concepto que ha sido abordado de diferentes formas por varios autores, por lo que no existe un consenso, aunque se ha propuesto como un constructo multidimensional compuesto por diversos componentes o atributos del servicio, por tanto es un juicio subjetivo de valoración en relevancia con el nivel de satisfacción de las personas usuarias finales. No obstante mencionan:

“[...] que una de las definiciones más aceptadas es la de Parasuraman, Zeithaml y Berry (1988), quienes la definen como "[...] una forma de actitud, relacionada pero no equivalente a la satisfacción, y resulta de la comparación de las expectativas con la percepción de desempeño" . Esta definición ha sido la base para muchos otros estudios (Vera \& Trujillo León, 2017). 
Conocer la opinión de las personas usuarias sobre los productos y servicio que reciben, resulta fundamental para las empresas, incluyendo los SA; puesto que, al satisfacer las necesidades y expectativas de estos, es posible construir opiniones positivas, que son el fin de la calidad de servicio. Es por esta razón, que resulta de suma importancia recolectar información de manera continua sobre lo que esperan las personas usuarias sobre un producto o servicio (Palacios Palacios, 2014).

\section{Perfil del usuario}

De la muestra de 409 participantes, se obtuvo 391 cuestionarios completos, de los cuales el 84.7\% eran estudiantes y el 81.5\% eran menores de 30 años lo cual era de esperar al estar en un entorno universitario, como se aprecia en el cuadro 1.

En relación a la ocupación de los participantes, el 84,7\% son estudiantes de la UCR, y con respecto a la situación laboral el 70,1\% se dedican al sólo al estudio, el 13,6\% trabaja y estudia. El $15,3 \%$ restante de los participantes representa a funcionarios de la universidad u otros (pensionados en cursos libres o público en general).

Cabe destacar que de los participantes el 44,5\% indicaron asistir a los comedores de 2-3 días/semana. Los ambientes universitarios no escapan de esta realidad, donde cada vez es mayor la demanda tanto de estudiantes como funcionarios, quienes son personas usuarias potenciales para los SA ubicados dentro y fuera de los campus universitarios (Joung et al., 2014). Esta población es generalmente muy diversa, $y$ los operadores de los SA se enfrentan al reto de satisfacer sus necesidades diariamente (Nadzirah et al., 2013), por lo que en estos casos la evaluación periódica de la calidad del servicio puede resultar en una herramienta muy útil para la mejora continua.

\section{CUADRO 1 \\ PERFIL DE USUARIO DE LOS COMEDORES SEGÚN FRECUENCIA DE ASISTENCIA. UNIVERSIDAD DE COSTA RICA, 2016 N=391}

\begin{tabular}{|c|c|c|c|c|c|c|c|c|}
\hline \multirow[b]{2}{*}{$\mathrm{N}$} & \multicolumn{2}{|c|}{ Total } & \multicolumn{2}{|c|}{1 día/semana } & \multicolumn{2}{|c|}{ 2-3 días/semana } & \multicolumn{2}{|c|}{$\begin{array}{c}4 \text {-5 días/ } \\
\text { semana }\end{array}$} \\
\hline & $\mathrm{N}$ & $\%$ & $\mathrm{n}$ & $\%$ & $\mathrm{n}$ & $\%$ & $\mathrm{n}$ & $\%$ \\
\hline \multicolumn{9}{|l|}{ Estudiante UCR } \\
\hline \multicolumn{9}{|l|}{ Edad } \\
\hline 17-20 años & 180 & 46.06 & 63 & 16.10 & 83 & 21.25 & 34 & 8.72 \\
\hline $21-29$ años & 139 & 35.57 & 50 & 12.78 & 69 & 17.66 & 20 & 5.13 \\
\hline 30-40 años & 7 & 1.79 & 3 & 0.77 & 5 & 1.28 & 1 & 0.26 \\
\hline 41 años o más & 5 & 1.28 & 1 & 0.26 & 2 & 0.51 & & \\
\hline \multicolumn{9}{|l|}{ Situación Laboral } \\
\hline Estudia y trabaja & 53 & 13.56 & 21 & 5.37 & 24 & 6.14 & 8 & 2.05 \\
\hline Sólo estudia & 278 & 71.14 & 96 & 24.53 & 135 & 34.56 & 47 & 12.05 \\
\hline Sub total Estudiante & 331 & 84.70 & 117 & 29.90 & 159 & 40.70 & 55 & 14.10 \\
\hline \multicolumn{9}{|l|}{ Funcionario UCR } \\
\hline \multicolumn{9}{|l|}{ Edad } \\
\hline 17-20 años & 2 & 0.77 & 1 & 0.46 & 1 & 0.35 & & - \\
\hline 21-29 años & 11 & 4.21 & 1 & 0.46 & 6 & 2.07 & 4 & 1.25 \\
\hline 30 años o más & 10 & 3.83 & 3 & 1.38 & 2 & 0.69 & 5 & 1.57 \\
\hline
\end{tabular}




\begin{tabular}{|c|c|c|c|c|c|c|c|c|}
\hline \multirow[b]{2}{*}{$\mathrm{N}$} & \multicolumn{2}{|c|}{ Total } & \multicolumn{2}{|c|}{1 día/semana } & \multicolumn{2}{|c|}{ 2-3 días/semana } & \multicolumn{2}{|c|}{$\begin{array}{l}4 \text {-5 días/ } \\
\text { semana }\end{array}$} \\
\hline & $\mathrm{N}$ & $\%$ & $\mathrm{n}$ & $\%$ & $\mathrm{n}$ & $\%$ & n & $\%$ \\
\hline 41 años o más & 17 & 6.50 & 5 & 2.30 & 2 & 0.69 & 13 & 4.08 \\
\hline \multicolumn{9}{|l|}{ Situación Laboral } \\
\hline Estudia y trabaja & 9 & 3.44 & 4 & 1.84 & 2 & 0.69 & 3 & 0.94 \\
\hline Trabaja & 30 & 11.48 & 6 & 2.76 & 9 & 3.11 & 18 & 5.65 \\
\hline Sub total Funcionario & 40 & 15.30 & 10 & 4.60 & 11 & 3.80 & 22 & 6.90 \\
\hline \multicolumn{9}{|l|}{ Otros } \\
\hline \multicolumn{9}{|l|}{ Edad } \\
\hline 17-20 años & 1 & 0.38 & - & - & 1 & 0.35 & - & - \\
\hline 21-29 años & 4 & 1.53 & 2 & 0.92 & 1 & 0.35 & - & - \\
\hline 30 años o más & - & - & - & - & - & - & - & - \\
\hline 41 años o más & 3 & 1.15 & - & - & 2 & 0.69 & 5 & 1.57 \\
\hline Situación Laboral & - & - & - & - & - & - & - & - \\
\hline Estudia y trabaja & 1 & 0.38 & - & - & - & - & - & - \\
\hline Sólo estudia & 15 & 5.74 & 4 & 1.84 & 4 & 1.38 & 6 & 1.88 \\
\hline Otro & 4 & 1.53 & 4 & 1.84 & - & - & - & - \\
\hline Sub total Otros & 20 & 7.65 & 8 & 3.68 & 4 & 1.38 & 5 & 1.57 \\
\hline Total General & 391 & 100.00 & 135 & 34.50 & 174 & 44.50 & 82 & 21.10 \\
\hline
\end{tabular}

Fuente: Elaboración Propia

\section{Dimensiones de la calidad de servicio}

En un SA las personas usuarias buscan la calidad, de modo que resulta importante tener clara su definición, puesto que de esta dependerá la experiencia que vayan a percibir y depende de múltiples criterios, de los cuales es de especial interés la experiencia de usuario (Romero \& López, 2013). Las experiencias son comprendidas como eventos propios de cada individuo, puesto que el consumidor comienza a buscar experiencias significativas en todos sus actos de consumo, privilegiando los aspectos subjetivos, en lugar de basarse exclusivamente en los aspectos objetivos y utilitarios asociados a productos y servicios (Gimenes et al., 2012).

En el gráfico 1 se muestra cierta relación entre la edad y la ocupación con respecto al puntaje otorgado a cada dimensión; donde se percibe diferencias que se van a ampliar en cada apartado. 


\section{GRÁFICO 1 \\ RELACIÓN DE EDAD Y OCUPACIÓN DEL USUARIO SEGÚN DIMENSIONES DE CALIDAD DE SERVICIO DE LOS COMEDORES DE LA UNIVERSIDAD DE COSTA RICA, 2016}
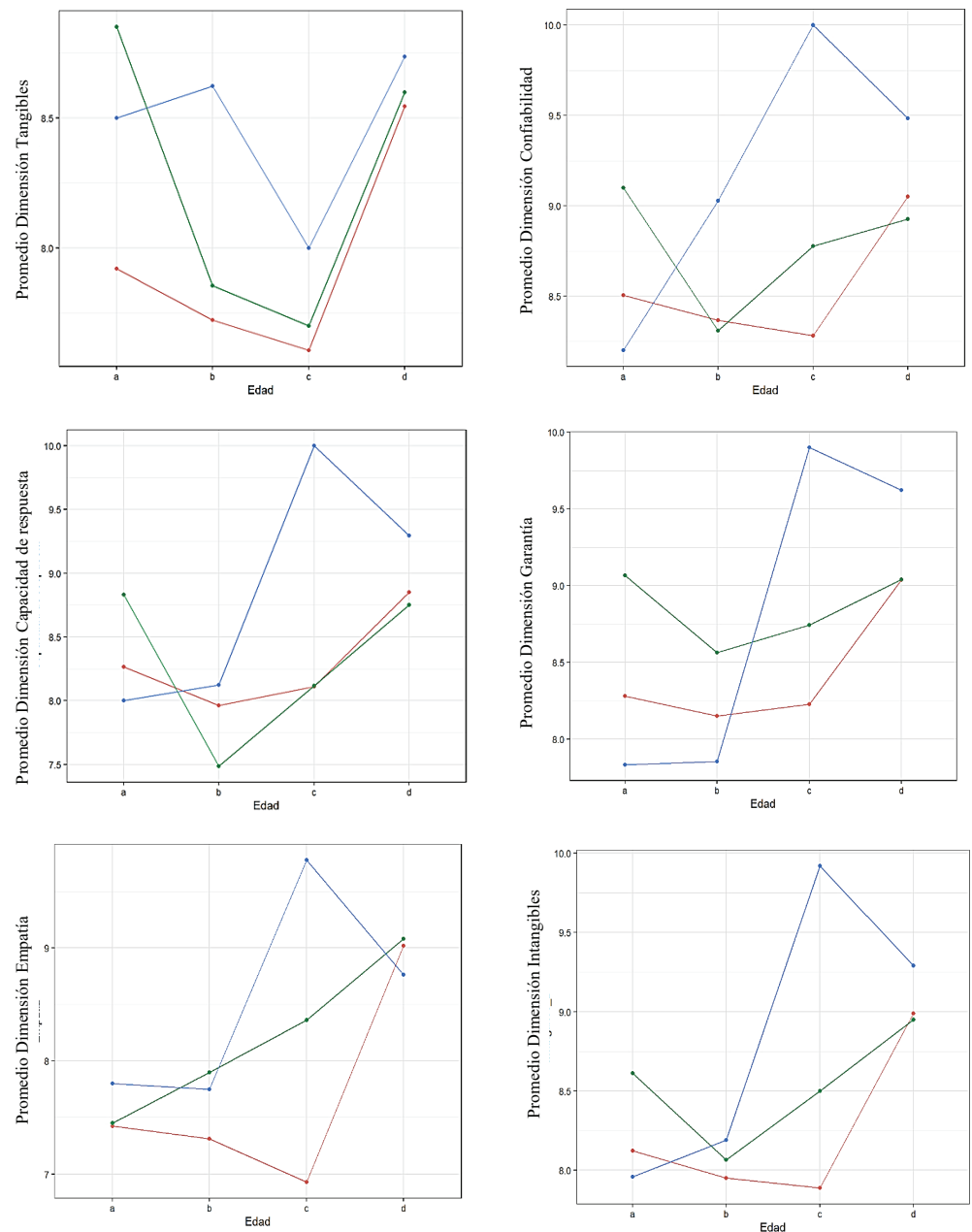

Ocupación Estudiante

Funcionario

Otros

Fuente: Elaboración Propia

Asimismo se realizarán pruebas inferenciales para poder medir la significancia de los mismos. Los resultados de la evaluación para cada una de las dimensiones de la calidad y los atributos que las componen según la opinión de las personas usuarias se presentan en el anexo 2 . A continuación se presentan lo más relevante encontrado en cada una de las dimensiones de calidad de servicio del estudio.

\section{Dimensión tangible}

Dicha dimensión responde a una serie de atributos principalmente relacionados a la infraestructura con la que cuentan algunos de los SA, las áreas, la presentación personal y la apariencia del lugar y del menú según el usuario. Son los aspectos físicos que el cliente percibe, 
tales como el diseño físico área del comedor, la apariencia del personal de contacto y la limpieza del lugar. En esta dimensión se omitió la pregunta de la limpieza de los baños puesto que dicha responsabilidad no recae en el SA de forma directa en este caso, los resultados se muestran en el gráfico 2.

\section{GRÁFICO 2 \\ PUNTAJE PROMEDIO ASIGNADO A LA DIMENSIÓN TANGIBLES DEL SA SEGÚN PERFIL DEL USUARIO. UNIVERSIDAD DE COSTA RICA, 2016}
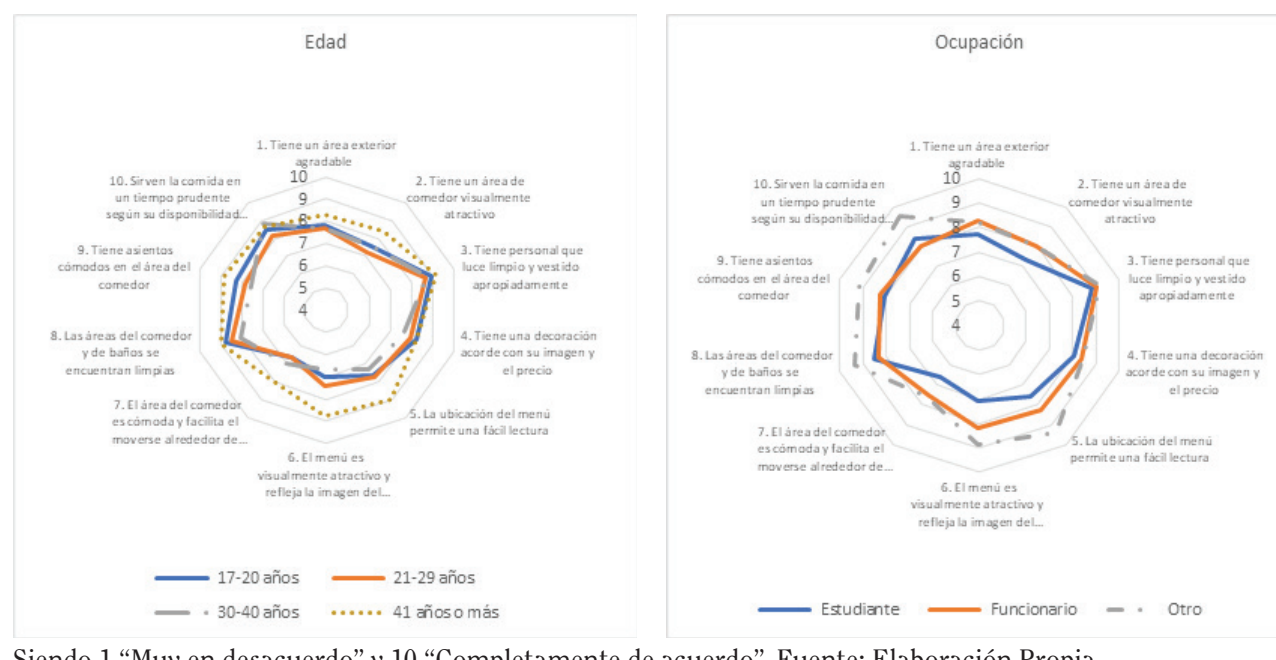

Siendo 1 "Muy en desacuerdo" y 10 "Completamente de acuerdo", Fuente: Elaboración Propia Kruskal-Wallis Tangibles, Edad $\mathrm{P}=0.00441^{*}$; Tangibles, Ocupación $\mathrm{P}=0.01106$ *

La dimensión de tangibles fue evaluada por el total de los participantes con un puntaje de 7,9 el cual se considera bajo con respecto a lo esperado en esta dimensión; puesto que se puede observar que a pesar de que los ítems relacionados a que el personal luce limpio y vestido apropiadamente y las áreas del comedor se encuentran limpias,(8,9 y 8,5), el aspecto relacionado con la comodidad del espacio y la facilidad para moverse (6.6) afectó el puntaje general, lo anterior puesto que en general es espacio del comedor de los diferentes SA es pequeño, en especial en horarios de mayor afluencia.

En lo que respecta al espacio disponible en el área del comedor, la comodidad y facilidad de moverse alrededor de ella, los puntajes generales fueron bajos 7,4 y 6,8 respectivamente, siendo aún menor en lo otorgado por los estudiantes respecto a los otros segmentos de usuarios. Lo anterior, no es adecuado en los SA participantes a pesar que la normativa nacional vigente regida por el Decreto No. 37.308-S. Reglamento de los Servicios de alimentación al público del Ministerio de Salud señala que las diferentes áreas de los SA al público deben de disponer de espacio suficiente para cumplir satisfactoriamente con todas las operaciones, dentro de las cuales se incluye la facilidad y comodidad para servir los alimentos y la circulación de las personas usuarias en el área de consumo (Presidencia de la República de Costa Rica, 2012) es una oportunidad de mejora que deberá involucrar decisiones institucionales puesto que se relaciona con las características de la infraestructura existentes.

En lo referente a los alrededores de un SA el reglamento indica que se deben mantener en buenas condiciones, no solo para evitar el ingreso de insectos, roedores y otras plagas, sino también que sea agradable a la vista de las personas usuarias (Presidencia de la República de Costa Rica, 2012). Los concesionarios se comprometen contractualmente cumplir con procedimientos 
y técnicas de limpieza, desinfección y saneamiento, los cuales son verificados por la OSG, con el fin de prevenir la contaminación de los alimentos y la afectación de la salud de las personas usuarias, basados en buenas prácticas de manufactura y manipulación de alimentos (Oficina de Suministros, 2019).

En el caso de la presentación personal de los colaboradores, se observa como la mayoría están de acuerdo con que estos lucen limpios (8,5 con 1,5 DS) y vestidos apropiadamente (8,9 con 1,4 DS). Estos resultados demuestran cómo los administradores cumplen con los lineamientos del cartel de la licitación pública, donde se indica que el contratista debe suministrar a su personal un uniforme completo de color blanco o claro, el cual debe mantenerse limpio durante las labores, e incluir redecilla que cubra todo el cabello, gabacha o delantal blanco, zapatos cerrados y guantes para manipular alimentos, lo cual da una presentación personal ideal a los colaboradores del servicio (Oficina de Suministros, 2019).

Siguiendo las pruebas post hoc del test Kruskal-Wallis de la dimensión tangible de $\mathrm{P}=$ 0.00441 para edad y P 0.01106 para ocupación. Respecto de los grupo de edad se obtiene que aunque ambas variables presentan diferencias, es la edad donde presentan diferencias significativas en los puntajes otorgados por los mayores de 41 años con los grupos de edades de 17-20 años y de 21-29 años al 95\% de confianza según la prueba de Nemenyi de pares extendidos, $\mathrm{P}=0.0175$ y $\mathrm{P}=0.0077$ respectivamente, no obstante por el contrario en la ocupación no se logra encontrar una diferencia entre pares.

\section{Dimensión confiabilidad}

La dimensión de confiabilidad está conformada por cinco apartados, los cuales son los siguientes: corrigen de forma inmediata cualquier error en el servicio, es consistente en el servicio que brinda, el cobro se realiza en forma rápida y sin errores y sirven la comida en forma rápida y sin errores y el personal se ayudan para mantener velocidad y calidad del servicio. Esta dimensión se encuentra en segundo mejor lugar con un promedio de 8,53 de las calificaciones totales con una desviación estándar de 1,47, tal como se observa en el gráfico 3.

\section{GRÁFICO 3 \\ PUNTAJE PROMEDIO ASIGNADO A LA DIMENSIÓN DE CONFIABILIDAD DEL SA SEGÚN PERFIL DEL USUARIO. UNIVERSIDAD DE COSTA RICA, 2016}

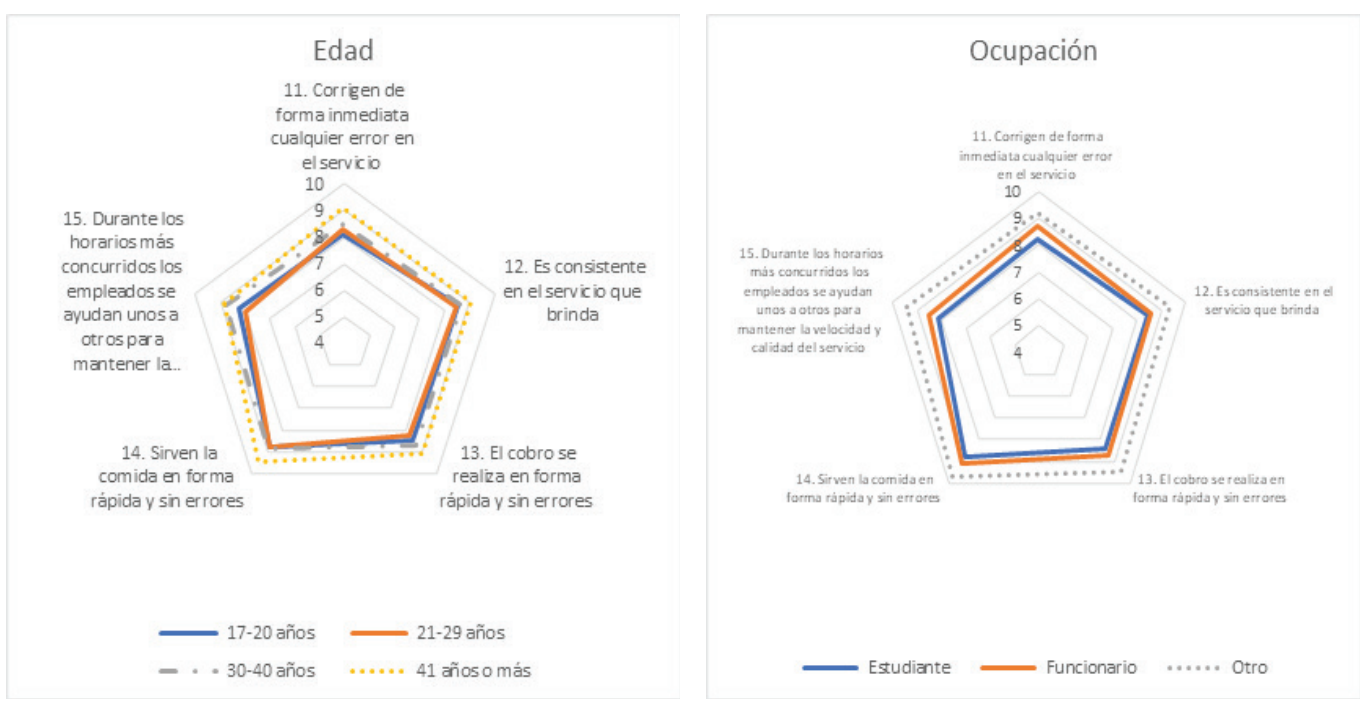

Siendo 1 "Muy en desacuerdo" y 10 "Completamente de acuerdo", Fuente: Elaboración Propia Kruskal-Wallis Confiabilidad, Edad P=0.01646; Confiabilidad, Ocupación P=0.002221 
En el gráfico 3 se muestran los resultados particulares para los atributos que conforman esta dimensión, siendo el de servir la comida de forma rápida y sin errores a la que se le asignó el mayor puntaje $(8,9)$ y esta opinión se mantiene constante en los diferentes rangos de edad y relación laboral. En la presente investigación la confiabilidad obtuvo la segunda mejor calificación para los SA, lo cual significa que las personas usuarias perciben un buen nivel de confiabilidad. Cabe destacar que en las pruebas Kruskal-Wallis indica diferencias en confiabilidad tanto para ocupación como para las diferentes edades del estudio, sin embargo esa diferencia es significativa entre los resultados de confiabilidad de los estudiantes con los otros externos a la universidad $(\mathrm{P}=0.012)$, lo que también se confirma que el grupo de 41 años o más presenta diferencias con el grupo de 21-29 años $\mathrm{P}=0.020$.

\section{Dimensión capacidad de respuesta}

La capacidad de respuesta se encuentra conformada por tres atributos, los cuales son: un servicio oportuno y rápido, y la realización de un esfuerzo extra por parte de los empleados para cumplir con las peticiones de las personas usuarias. En el gráfico 4 se presenta el promedio del puntaje obtenido de la evaluación para cada uno de los atributos de dicha dimensión.

\section{GRÁFICO 4 \\ PROMEDIO DEL PUNTAJE DE LAS PERSONAS USUARIAS OBTENIDOS POR ATRIBUTO CON RESPECTO A LA DIMENSIÓN CAPACIDAD DE RESPUESTA DEL SA SEGÚN PERFIL DEL USUARIO. UNIVERSIDAD DE COSTA RICA, 2016}

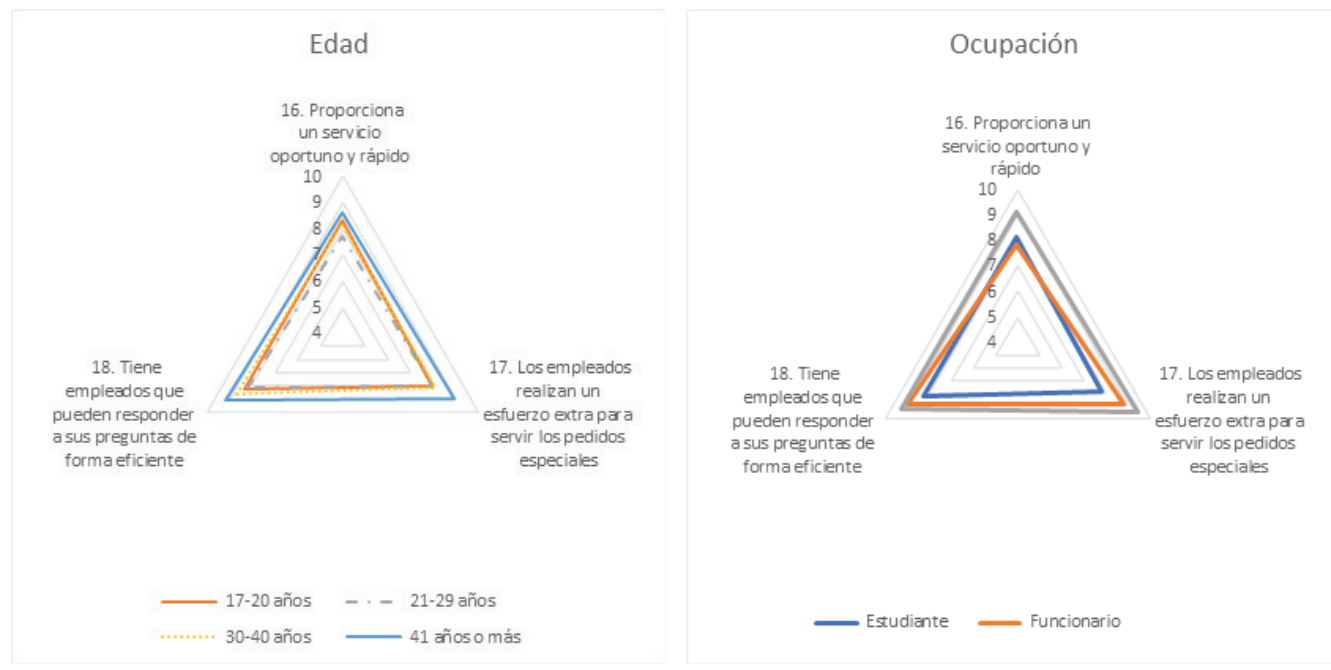

Siendo 1 "Muy en desacuerdo" y 10 "Completamente de acuerdo",

Kruskal-Wallis Confiabilidad, Edad $\mathrm{P}=0.01646$; Confiabilidad, Ocupación $\mathrm{P}=0.002221$

Fuente: Elaboración Propia 
A pesar de que los puntajes generales son satisfactorios, la capacidad de respuesta es vital para tomar en cuenta en los SA de tipo universitario, donde el servicio debe ser rápido, se asignó 8,1.

Por su parte los estudiantes que contestaron, estos asignaron el puntaje de 7,9 en comparación a los otros atributos de esta dimensión. Asimismo se observa que los encuestados de edades entre 21 a 29 años, tienen los puntajes más bajos. Siendo la capacidad de respuesta en la pregunta de que debe existir de parte del personal del SA una buena disposición hacia las personas usuarias y ofrecer un servicio rápido, debe tomarse en cuenta como oportunidad de mejora.

En este caso es fundamental revisar si existen diferencias entre las variables para poder orientar la toma de decisiones, nuevamente se presenta diferencia entre las edades de 41 años o más con los de 21-29 años $\mathrm{P}=0.0067$, por lo que es importante plantear estrategias diversas para brindar la respuesta que requieren ambas poblaciones.

En un estudio realizado para evaluar la calidad de servicio brindado por los mejores diez y más populares restaurantes en Taiwán, se obtuvo que entre las cinco dimensiones de calidad, la "capacidad de respuesta" fue la más importante (Tzeng \& Chang, 2011), lo anterior es positivo en especial puesto que a pesar de ser comedores universitarios y no restaurantes la evaluación fue adecuada.

En la capacidad de respuesta es fundamental que exista la colaboración necesaria para retomar algún trabajo que haya quedado mal realizado por alguno de los miembros de la cadena interna de servicio (Hollis, 2006). Para el atributo acerca de la colaboración necesaria el puntaje promedio de 8,1 (DS 1,8), reflejando que los resultados en su mayoría fueron bastante positivos, lo que indica que durante los horarios más concurridos se mantiene la velocidad y calidad del servicio debido a la colaboración que existe entre los empleados.

\section{Dimensión garantía}

La dimensión garantía se compone por cinco atributos: el trato del personal lo hace sentir bien y cómodo, los empleados tienen el conocimiento para contestar todas las preguntas sobre el platillo que sirven en materia de ingredientes y forma de prepararlos, lo hicieron sentirse seguro en todo momento, el personal es capacitado, competente y experimentado, se sintió que la soda/ comedor apoya al personal para que haga bien su trabajo y si notó que el personal es sensible a sus necesidades en lugar de sólo seguir políticas y reglas, para los cuales se presenta el promedio obtenido seguidamente del gráfico 5 .

De las cinco dimensiones, garantía recibió el segundo lugar del puntaje en general con un $8,35, y$ esto es adecuado para considerar puesto que en el caso de Guzmán López y Cárcamo Solís en 2014, la dimensión garantía en su estudio fue la mejor calificada, afirman que las personas usuarias perciben el conocimiento y cortesía por parte del personal de los SA, lo cual genera confianza y seguridad a la hora de asistir y adquirir los productos y servicios de estos establecimientos (Guzmán López, \& Cárcamo Solís, 2014).

Estos resultados deben de ser considerados al momento de plantearse estrategias relacionadas con la calidad de servicio ofrecida en los SA universitarios, puesto que es necesario, que se mantengan los factores que generan estos sentimientos positivos, relacionados a que el usuario sea capaz de: confiar en las recomendaciones del personal y tener la seguridad de que los alimentos y/o bebidas están preparados sin contaminación alguna; así como poder expresar alguna preocupación, inquietud o pregunta sin temor alguno tal como menciona también Guzmán López y Cárcamo Solís en 2014. 


\section{GRÁFICO 5 \\ PROMEDIO ASIGNADO A LA DIMENSIÓN GARANTÍA DEL SA SEGÚN PERFIL DEL USUARIO. UNIVERSIDAD DE COSTA RICA, 2016}

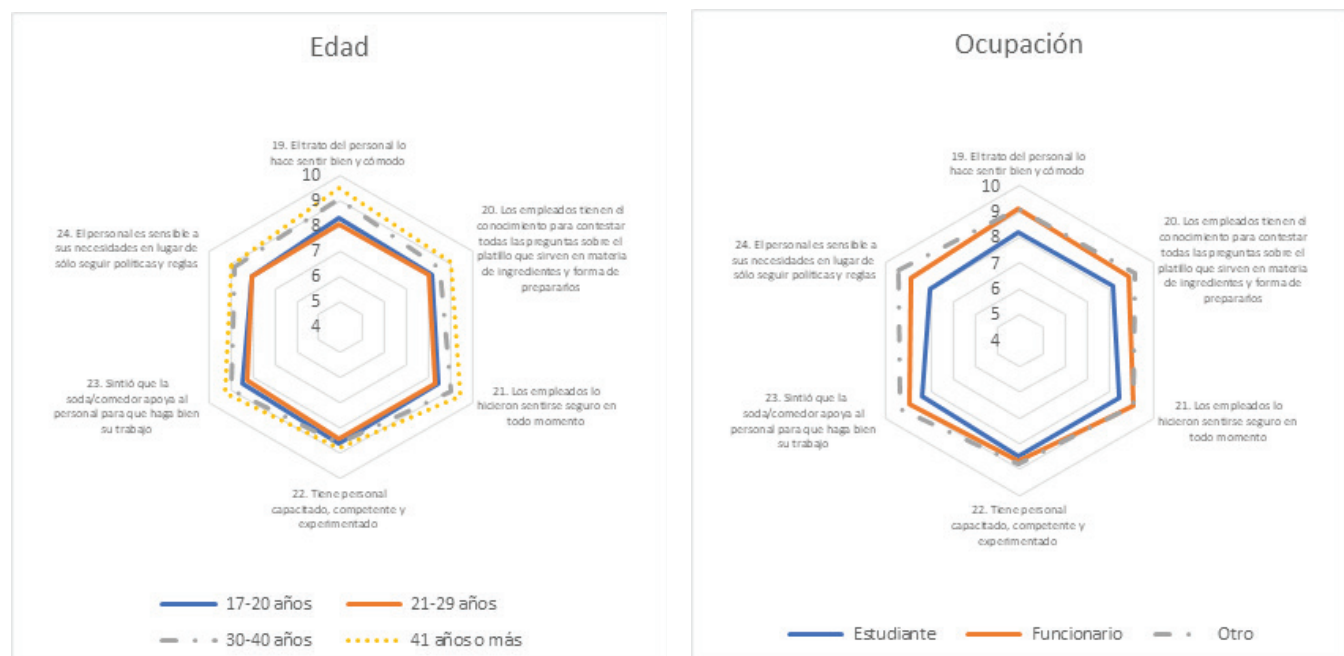

Siendo 1 "Muy en desacuerdo" y 10 "Completamente de acuerdo",

Kruskal-Wallis Confiabilidad, Edad $\mathrm{P}=0.01646$; Confiabilidad, Ocupación $\mathrm{P}=0.002221$

Fuente: Elaboración Propia

Como se puede observar en el gráfico 5, las respuestas de la dimensión garantía se ubicaron entre 8,6 - 8,3, siendo mayor el atributo de tiene que ver con tener personal capacitado, competente y experimentado $y$ las preguntas de que los empleados tienen el conocimiento para contestar todas las preguntas sobre el platillo que sirven en materia de ingredientes $y$ forma de prepararlos el atributo el puntaje menor. Según la ocupación de las personas usuarias se analizó si el trato del personal les hizo sentir bien y cómodos, $y$ en este caso el mayor puntaje fue asignado por los funcionarios.

A pesar de las buenas calificaciones, ninguno de los atributos de esta dimensión alcanza a cumplir con el 100\% de satisfacción de las expectativas de sus usuarios, siendo el trato del personal y que este sea sensible a sus necesidades son los atributos que más se alejan de esa meta, convirtiéndose en un punto de mejora en cada uno de los SA.

Una de las principales razones de brindar la mejor atención del personal está directamente relacionada con un adecuado o deficiente servicio al cliente, el cual es un elemento fundamental $y$ tan poderoso como los descuentos o la publicidad a la hora de generar ventas, $y$ de obtener $y$ mantener usuarios (Lira Mejía, 2009).

La situación anterior debe ser tomada en cuenta por los administradores de los SA al momento de capacitar al personal y de definir indicadores relacionados con el trato hacia las personas usuarias, ya que se debe tener presente todas las características y necesidades de la población mayoritaria a la que se está dando el servicio, puesto que de esta depende tanto la evaluación del servicio ofrecido.

En la prueba post hoc en cuanto a edad se muestra que el grupo de 41 años o más es diferente respecto del grupo de 17-20 años y el grupo 21-29 años ( $\mathrm{P}=0.00054$ y $\mathrm{P}=0.00037$ respectivamente). Y cabe destacar que en garantía si se muestra una diferencia significativa por 
ocupación de la opinión de los Estudiantes con respecto a los otros grupos del estudio.(Funcionario $\mathrm{P}=0.0024$ y Otros $\mathrm{P}=0.0027$ ); por lo que los planes de mejora deben contemplar objetivos diferentes.

Debido al peso de la dimensión de Garantías, la capacitación del personal de contacto en los servicios de alimentación, es de vital importancia en la evaluación de la calidad de los servicios de alimentación por lo que la formación y capacitación de los trabajadores es necesaria, que incluso en las normativas internacionales existen apartados exclusivos relacionados con este tema, por ejemplo en el apartado 6.2 de la norma ISO 9001-2008 detalla que para satisfacer a las personas usuarias, las organizaciones además de productos de calidad, deben tener personal capacitado que cuente con requisitos de educación, formación, habilidades y experiencia para cada puesto de trabajo (Vinca, 2011).

\section{Dimensión empatía}

Cuando se habla de la dimensión empatía se relaciona a todo lo concerniente a la capacidad de brindar una atención personalizada a los clientes mediante la previsión de necesidades (dietéticas, especiales) o por la disposición del personal de ser comprensivo hacia las necesidades de los clientes (Guzmán López, \& Cárcamo Solís, 2014).

Dentro de esta dimensión, uno de los atributos evaluados fue si le hicieron sentir como alguien especial, lo que resultó en una puntuación de 7. En el caso de si el personal es sensible a sus necesidades en lugar de sólo seguir políticas y reglas; este ítem obtuvo mayor porcentaje lo cual es contradictorio con respecto al anterior y podría indicar problemas de comprensión del ítem. Este fenómeno se refleja en los resultados obtenidos, donde la dimensión de empatía (un aspecto intangible) obtuvo la calificación más baja por parte de las personas usuarias, tal como se muestra en el gráfico 6 .

\section{GRÁFICO 5 \\ PROMEDIO ASIGNADO A LA DIMENSIÓN GARANTÍA DEL SA SEGÚN PERFIL DEL USUARIO. UNIVERSIDAD DE COSTA RICA, 2016}
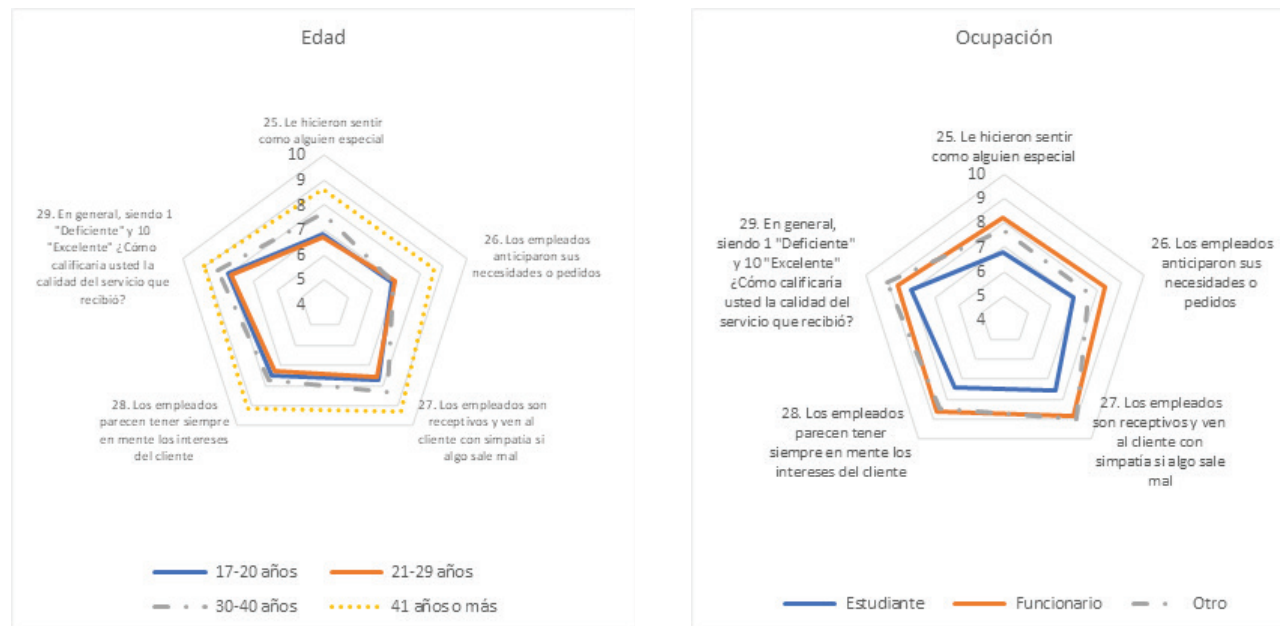

Siendo 1 "Muy en desacuerdo" y 10 "Completamente de acuerdo"

Kruskal-Wallis

Empatía, Edad P=1.037e-06; Empatía, Ocupación P=4.558e-07

Fuente: Elaboración Propia 
En lo que respecta a la dimensión empatía, esta se compone de aspectos como la sensibilidad del personal hacia las necesidades e intereses de las personas usuarias, además de la receptividad, simpatía, atención y la anticipación a sus peticiones. Según Hassan y Mousa (2012) esta dimensión se engloba en tres grupos importantes la accesibilidad, comunicación y comprensión hacia el usuario.

$\mathrm{Al}$ ser el usuario tan diverso, es clave revisar si existen diferencias en cuanto al puntaje dado a esta dimensión para así orientar las estrategias de mejora. Al medir la prueba post hoc el test de Nemenyi que son diferentes el grupo de 41 años o más respecto del 17-20 años. ( $\mathrm{P}=0.000005)$ y el 21-29 años $(\mathrm{P}=0.0000041)$. Cabe destacar que esta también muestra una diferencia significativa por ocupación de la opinión del grupo estudiantes con respecto a los funcionarios y los otros $(\mathrm{P}=0.000019$ y $\mathrm{P}=0.0093$ respectivamente)

La empatía puede definirse como la capacidad de percibir correctamente lo que experimenta otra persona, y comunicar esta percepción en un lenguaje acomodado a sus sentimientos (Bermejo Higuera et al., 2013). Además, está formada por dos componentes, uno de tipo cognitivo, donde se entiende la empatía como la capacidad de ponerse intelectualmente en el lugar del otro y comprender sus emociones; $y$ otro componente afectivo donde se logra sintonizar emocionalmente con otra persona (López Pérez, \& Fernández Pinto, 2010). Por lo que es determinante que los SA en estudio presten atención y dirijan esfuerzos a reforzar los aspectos calificados positivamente y tomar medidas en mejorar los deficientes.

\section{Modelo de regresión lineal múltiple de las dimensiones de la calidad de servicio}

Es posible notar cómo la literatura existente hace referencia, en mayor medida, a calidad de servicio en SA comerciales; no obstante, existen países como Malasia que se interesan en el tema de calidad en SA, por ejemplo este país, exige a las universidades ofrecer los mejores productos y servicios a sus clientes potenciales del campus, principalmente los estudiantes. Por ello, los operadores de servicios de alimentación universitarios luchan continuamente para complacer a las comunidades universitarias que son naturalmente diversas y dinámicas (Othman et al., 2013).

En los anteriores apartados se ha detallado lo encontrado en cada dimensión, Asimismo, con respecto a la calificación general de la calidad del servicio recibido en los SA universitarios, esta fue de 8,07 con una desviación estándar de 1,49, con lo que se puede orientar a la toma de decisiones $y$ planes de mejora en los aspectos que lo merecen no obstante para poder determinar la relación existente entre la calidad y las dimensiones del instrumento utilizado se realizó un modelo de regresión lineal múltiple presentados en el cuadro 2. 


\section{CUADRO 2 \\ MODELO DE REGRESIÓN LINEAL MÚLTIPLE DE LA CONTRIBUCIÓN DE LAS DIMENSIONES DE CALIDAD DE SERVICIO DE LOS SA UNIVERSITARIOS. SEDE RODRIGO FACIO. UNIVERSIDAD DE COSTA RICA, 2016}

\begin{tabular}{lcccccc}
\hline \multicolumn{1}{c}{ Coeficientes: } & Beta & $\begin{array}{c}\text { Beta } \\
\text { estandarizado }\end{array}$ & Std. Error & $\mathrm{t}$ value & $\operatorname{Pr}(>|\mathrm{t}|)$ & \\
\hline Promedio & 5.35351 & -0.030627 & 2.53527 & 2.112 & 0.035369 & $*$ \\
$21-29$ años & 0.07336 & 0.003492 & 1.31279 & 0.056 & 0.955468 & \\
$30-40$ años & 3.69585 & 0.175913 & 3.04274 & 1.215 & 0.225252 & \\
41 años o más & 5.28436 & 0.251522 & 2.71422 & 1.947 & 0.052277 & $*$ \\
Funcionario UCR & -0.5378 & -0.025598 & 2.48464 & -0.216 & 0.828752 & \\
Otro & -0.68837 & -0.032764 & 3.39458 & -0.203 & 0.839412 & \\
Tangibles & 0.28048 & 0.257271 & $4.14 \mathrm{E}-02$ & 6.775 & $4.73 \mathrm{E}-11$ & $* * *$ \\
Confiabilidad & 0.17323 & 0.176257 & $5.12 \mathrm{E}-02$ & 3.383 & 0.000792 & $* * * *$ \\
Garantía & 0.17747 & 0.188145 & 0.05131 & 3.459 & 0.000603 & $* * * *$ \\
Empatía & 0.16266 & 0.216923 & $3.39 \mathrm{E}-02$ & 4.797 & $2.32 \mathrm{E}-06$ & $* * *$ \\
Capacidad de Respuesta & 0.10817 & 0.120546 & 0.04625 & 2.339 & 0.019847 & $*$ \\
\hline Realza & 0.025 & & & \\
\hline
\end{tabular}

Realizado transformación Box cox para normalizar los datos con un Lambda 2

Multiple R-squared: 0.6933 , Adjusted R-squared: 0.6853

F-statistic: 86.36 on 10 and $382 \mathrm{DF}$, p-value: $<2.2 \mathrm{e}-16$

En el cuadro 2 las edades se comparan con la edad 17-20 años y la ocupación se compara con el grupo de estudiantes para su análisis al ser nominales. Este se ajustó revisando cumplimiento de los supuestos y son independientes, normales y con varianzas iguales, por lo que se realizó la transformación. En la interpretación se tiene que en un modelo general ni la edad y ni la ocupación afectan la calidad, $y$ las dimensiones usadas en el instrumento efectivamente afectan la evaluación de la calidad de servicio total, siendo la que más afecta la dimensión tangible, seguido de garantía, confiabilidad y empatía, y el que menos la capacidad de respuesta respecto a los puntajes.

Se considera que el nivel de satisfacción del usuario es un buen indicador de la calidad de servicio, $y$ en un estudio realizado en Colombia, Concha (2010) interpreta que la confiabilidad y la capacidad de respuesta son sus principales componentes y esta se mantiene según los rangos de edad y ocupación, por lo que se observa que el servicio recibido es satisfactorio por las personas usuarias. No obstante la capacidad de respuesta puntuó con un beta menor, pero si significativo.

En la presente investigación se observó que la dimensión empatía obtuvo las menores calificaciones; es decir que las personas usuarias perciben no recibir un trato empático o personalizado, e inclusive en algunos casos consideran que este ni siquiera aplica para estos servicios, $y$ al ser este de las variables que se relacionan más con la calidad de servicio $\mathrm{P}=2.32 \mathrm{E}-06$, debe de ser tomada en cuenta en futuros planes de acción de los servicios de alimentación y para las nuevas contrataciones. La calidad del servicio general se ve influenciada fuertemente por una atención amable por parte del personal (Vanniarajan \& Meharajan, 2012), y es por esto que en todo SA, independientemente del tipo, se debe reforzar la importancia de capacitar al personal $y$ hacer un trato agradable al usuario arte de su servicio un trato agradable al usuario. 


\section{CONCLUSIONES}

Al identificar las dimensiones de calidad de servicio a partir de la experiencia del usuario que asiste a los servicios de alimentación del estudio, por medio del instrumento DINESERV que plantea cinco dimensiones que componen la calidad de servicio, correspondientes a tangibles, confiabilidad, capacidad de respuesta, garantía y empatía. Se encontró que las dimensiones de garantía y confiabilidad obtuvieron los mayores puntajes, mientras que tangibles y empatía resultaron con la menor puntuación; sin embargo de manera general las personas usuarias indicaron estar conformes con la calidad de servicio recibido puesto que el 74,57\% calificó entre excelente y bueno.

Las evaluaciones de las dimensiones de la calidad de servicio, muestran que la confiabilidad y la garantía obtuvieron los puntajes más altos 8,53 y 8,35 respectivamente, lo cual concuerda con la valoración general del usuario. Por su parte, la dimensión tangible y la empatía presentan menores puntajes. Estas cuatro dimensiones fueron las que puntuaron más alto en los resultados del método de regresión realizado, lo cual comprueba que son estas áreas de mejora prioritarias para ser tomadas en la toma de decisiones.

Al analizar la calidad de los servicios de alimentación de la Sede Rodrigo Facio de la Universidad de Costa Rica según la experiencia de las personas usuarias; se puede concluir que en general la calidad de servicio es aceptable con un promedio de 8,07 con una desviación estándar de 1,49 y esto puede ser sostenible en el tiempo pero que existen oportunidades de mejora que deben ser considerados a fin de aumentar el número de visitas a los SA universitarios y contar con la garantía de que la población estudiantil tiene una alimentación saludable.

Por lo anterior se recomienda incluir en los carteles de licitación lineamientos a seguir orientados a la calidad de servicio, $y$ en especial al servicio al cliente; $y$ que incluyan herramientas de evaluación permanente y capacitaciones que deben ser ofrecidas al personal.

Asimismo, se recomienda el uso del instrumento DINESERV como una herramienta de evaluación periódica de la calidad del servicio en los SA de la UCR por parte de la OSG, al menos una vez al año y que los resultados sean presentados a cada concesionario (encargado del SA) y sirvan para ajustar el cartel de licitación del siguiente periodo. Cabe recalcar que las diferencias pueden deberse a que cada comedor es administrado por diferentes concesionarios en diferentes instalaciones por lo que se debería estandarizar los servicios que ofrecen y mejorar el tamaño de los comedores. Este instrumento deberá adaptarse según sean las características de los servicios que se deseen evaluar para optimizar la comprensión y respuesta del usuario.

\section{AGRADECIMIENTOS}

Se les agradece por la colaboración a Katherine Angulo López, Tania Castro Ureña, Katherine Jiménez Murillo, Maricruz Mora Zamora, Katherine Villalobos Vega y María Fernanda Zamora Barahona por su participación. También a la Máster Marcela Peña Vásquez, nutricionista de la Sección de Servicios Contratados de la Oficina de Servicios. Asimismo a la PhD. Xinia Fernández-Rojas y al Ing. Pablo Zamora-Calvo por su apoyo estadístico.

Este artículo se realizó como parte del proyecto 450-B5-320: Experiencia en los comedores universitarios: Perspectivas de los usuarios de la Sede Rodrigo Facio, Universidad de Costa Rica con apoyo de la Vicerrectoría de Investigación y de la Escuela de Nutrición. El mismo fue aprobado por el Comité Ético Científico de la Universidad de Costa Rica, según consta en el acta núm. 282 en sesión del 08 de octubre 2014. 


\section{REFERENCIAS}

Aguirre, J., \& Rodríguez, M. (2011). Medir la Satisfacción de los clientes de restaurantes con el método DINESERV. Gaudeamus Revista Académica Universidad Latina, 3, 41-62.

Barrios, M. (2012). Marketing de la experiencia: Principales conceptos y características. Palermo Business Review, 7, 67-89.

Bermejo Higuera, J., Villacieros Durbán, M., \& Carabias Maza, R. (2013). Diseño y fiabilidad de una escala sobre la idea de empatía: Diferencias entre profesiones sanitarias y sociales. Acción Psicológica, 10(2), 143-156. https://doi.org/10.5944/ap.10.2.12217

Concha, J. (2010). Satisfacción del Cliente en Empresas de Servicio el Caso de la Industria de Restaurantes [Ponencia]. $1^{\circ}$ Simpósio Brasileiro de Ciência de Serĉos, Brasil. Universidad Icesi.

Espinoza, L., Rodríguez, R., Gálvez, J., \& MacMillan, N. (2011). Hábitos de alimentación y actividad física en estudiantes universitarios. Revista Chilena Nutrición, 38(4), 458-465. https://doi. org/10.4067/S0717-75182011000400009

García Bravo, V., \& López Sedó, V. (2015). Sodas del campus Rodrigo Facio alimentan a 5000 estudiantes cada día, Semanario Universidad. https://historico.semanariouniversidad.com/ universitarias/sodas-del-campus-rodrigo-facio-alimentan-a-5-000-estudiantes-cada-dia/

Gimenes, M., Fraiz, J., \& Gândara, J. (2012). Comidas inolvidables: La construcción de una metodología para analizar las experiencias de comer fuera de casa. Estudios y Perspectivas en Turismo, 21(4), 802-824. http://www.scielo.org.ar/scielo.php?script=sci_arttext\&pid $=\mathrm{S} 1851-17322012000400001$

Guzmán López, A., \& Cárcamo Solís, M. L. (2014). La evaluación de la calidad en el servicio: caso de estudio "Restaurant Familiar Los Fresnos". Acta Universitaria, 24(3), 35-49. https://doi. org/10.15174/au.2014.503

Hassan, T., \& Mousa, A. (2012). Measuring the Performance of Human Services Providers using SERVQUAL Model: Study on governmental hospitals services at Ministry of Health Khartoum state 2012. Journal of Natural and Medical Sciences, 14(2). http://repository. sustech.edu/handle/123456789/17255

Hollis, C. (2006). Service Quality Evaluation in Internal Healthcare Service Chains [Tesis de Doctorado, Queensland University of Technology]. https://eprints.qut.edu.au/16267/

Joung, H. W., Lee, D. S., Kim, H. S., \& Huffman, L. (2014). Evaluation of the on-campus dining services using importance-performance analysis. Journal of Foodservice Business Research, 17(2), 136-146. https://doi.org/10.1080/15378020.2014.902647

Kwun, D. J.-W. (2011). Effects of campus foodservice attributes on perceived value, satisfaction, and consumer attitude: A gender-difference approach. International Journal of Hospitality Management, 30(2), 252-261. https://doi.org/10.1016/j.ijhm.2010.09.001

Lee, Y. L., \& Hing, N. (1995). Measuring quality in restaurant operations: an application of the SERVQUAL instrument. International Journal of Hospitality Management, 14(3/4), 293-310. https://doi.org/10.1016/0278-4319(95)00037-2

Lira Mejía, C. (2009). ¿Cómo puedo mejorar el servicio a cliente? Técnicas para perfeccionar la actitud en el servicio a clientes. México.

López Pérez, B., \& Fernández Pinto, I. (2010). Diferencias de edad en empatía: desde la adolescencia hasta la tercera edad. Ansiedad y Estrés, 16(2), 139-150.

Mejías, A., Torres, E., \& Veloz, Y. (2012). Impacto de la Calidad de Servicio sobre la Satisfacción del Cliente: un estudio de caso en un comedor universitario. Revista Copérnico, 8(16). http:// copernico.uneg.edu.ve/numeros/c16/c16.html 
Nadzirah, S., Ab Karim, S., Ghazali, H., \& Othman, M. (2013). University foodservice: An overview of factors influencing the customers' dining choice. International Food Research Journal, 20(3), 1459-1468. http://www.ifrj.upm.edu.my/volume-20-2013.html

Oficina de Servicios Generales. (2012). Servicios de alimentación: UCR. Universidad de Costa Rica. https://web.archive.org/web/20191003030728/http://www.osg.ucr.ac.cr/index.php/os/2013-0111-22-33-25/130-servicios-de-alimentacion

Oficina de Suministros. (2019). Licitación Pública para la concesión temporal de un local para la prestación de servicios de alimentación. Universidad de Costa Rica. http://osum.ucr.ac.cr/ contrataciones/publicas

Othman, M., Salehuddin, N., Karim, M., \& Ghazali, H. (2013). Customers' Satisfaction towards Institutional Foodservices: An Insight into Universities in the Klang Valley, Malaysia. Journal of Business and Policy Research, 8(2), 1-15.

Palacios Palacios, D. (2014). Evaluación de la calidad de servicio percibida en los establecimientos hoteleros de Quibdó [Tesis de maestría, Universidad Nacional de Colombia]. UNAL. https:// repositorio.unal.edu.co/handle/unal/51538

Presidencia de la República de Costa Rica (2012). Decreto No. 37.308-S. Reglamento de los Servicios de alimentación al público. La Gaceta. http://www.pgrweb.go.cr/scij/Busqueda/Normativa/ Normas/nrm_texto_completo.aspx?param1=NRTC\&nValorl=1\&nValor2=5009\&nValor3=0\&s trTipM=TC

Ramírez, L. (2014). Concesión temporal de un local para la prestación de servicios de Licitación pública 2014LN-000011-UADQ Alimentación en la facultad de Educación. Universidad de Costa Rica.

Romero, I., \& López, V. (2013). La calidad del servicio en cafetería, según percepción de usuarios. Global Conference on Business and Finance Proceedings, 8(1).

Stevens, P., Knutson, B., \& Patton, M. (1995). DINESERV: A tool for measuring service quality in restaurants. Cornell Hotel and Restaurant Administration Quarterly, 36(2), 56-60. https:// doi.org/10.1177/001088049503600226

Tzeng, G., \& Chang, H. (2011). Applying Importance-Performance Analysis as a Service Quality Measure in Food Service Industry. Journal of Technology Management \& Innovation, 6(3) 106-115. https://doi.org/10.4067/S0718-27242011000300008

Universidad de Costa Rica (2019). Licitación pública 2018LN-000002-UADQ "Concesión temporal de un local para la prestación de Servicios de Alimentación en el comedor estudiantil". Vicerrectoría de Administración, Oficina de Suministros

Vanniarajan, T., \& Meharajan, T. (2012). Dineserv: A Tool for Measuring Service Quality in Restaurants. Journal of Marketing \& Communication 8(2), 4-11.

Vera, J., \& Trujillo León, A. (2017). Escala mexicana de calidad en el servicio en restaurantes (EMCASER). Innovar, 27(63), 43-60. https://doi.org/10.15446/innovar.v26n63.60665

Vinca (2011). Recursos Humanos. https://web.archive.org/web/20170502045417/http://www. normas9000.com/iso-9000-26.html 


\section{ANEXOS}

Anexo 1. Instrumento para la evaluación de la calidad de los servicios de alimentación de la Sede Rodrigo Facio de la UCR desde la experiencia de sus usuarios.

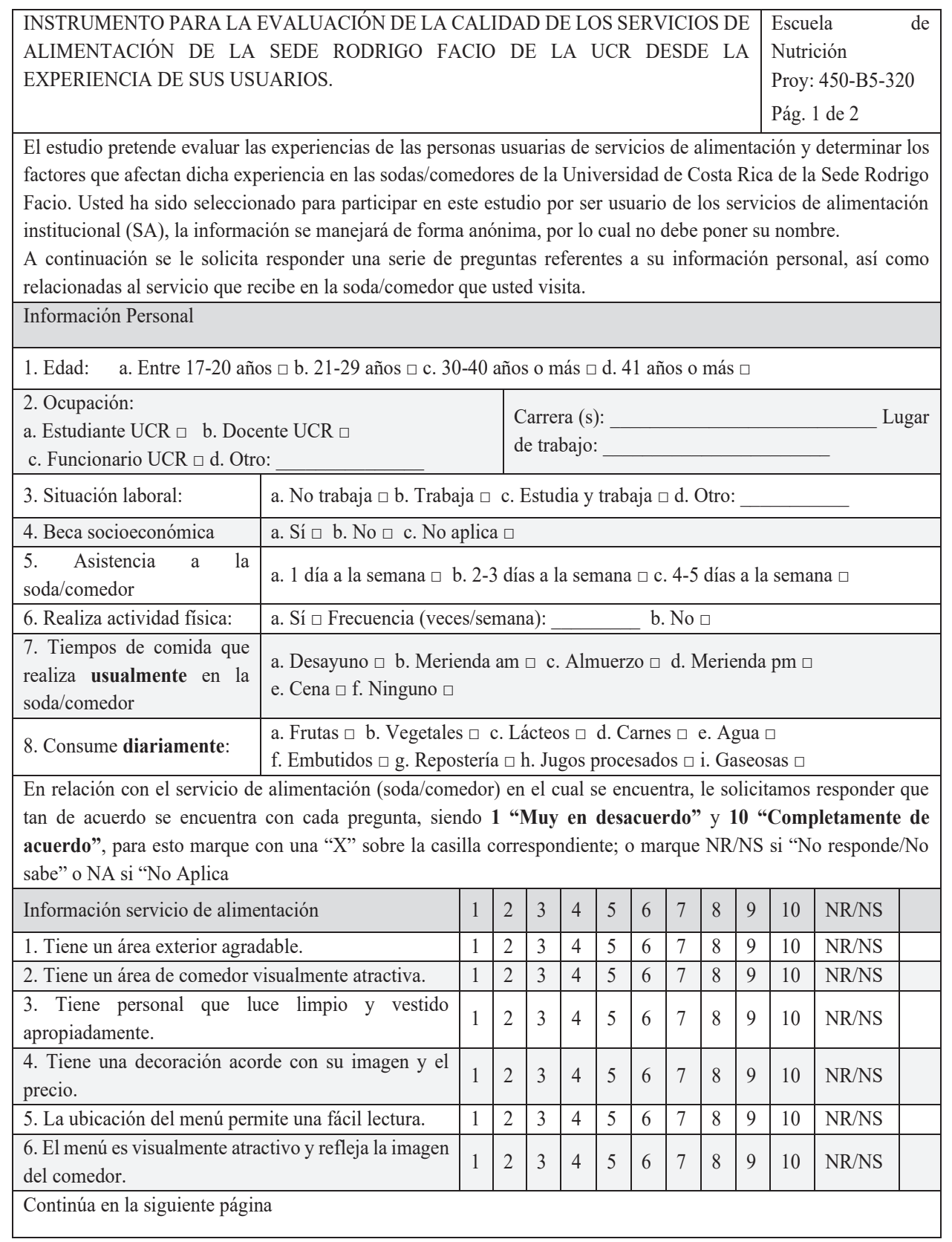




\begin{tabular}{|c|c|c|c|c|c|c|c|c|c|c|c|c|}
\hline \multicolumn{13}{|c|}{$\begin{array}{l}\text { Continuación: En relación con el servicio de alimentación (soda/comedor) en el cual se encuentra, le solicitamos } \\
\text { responder que tan de acuerdo se encuentra con cada pregunta, siendo } \mathbf{1} \text { "Muy en desacuerdo" y } \mathbf{1 0} \\
\text { "Completamente de acuerdo", para esto marque con una "X" sobre la casilla correspondiente; o marque NR/NS } \\
\text { si "No responde/No sabe" o NA si "No Aplica". }\end{array}$} \\
\hline Información servicio de alimentación & 1 & 2 & 3 & 4 & 5 & 6 & 7 & 8 & 9 & 10 & NR/NS & \\
\hline $\begin{array}{l}\text { 7. El área de comedor es cómoda y facilita el } \\
\text { moverse alrededor de ella. }\end{array}$ & 1 & 2 & 3 & 4 & 5 & 6 & 7 & 8 & 9 & 10 & $\mathrm{NR} / \mathrm{NS}$ & \\
\hline 8. Las áreas del comedor se encuentran limpias. & 1 & 2 & 3 & 4 & 5 & 6 & 7 & 8 & 9 & 10 & $\mathrm{NR} / \mathrm{NS}$ & \\
\hline 9. Tiene asientos cómodos en área de comedor. & 1 & 2 & 3 & 4 & 5 & 6 & 7 & 8 & 9 & 10 & $\mathrm{NR} / \mathrm{NS}$ & \\
\hline $\begin{array}{l}\text { 10. Sirven la comida en un tiempo prudente según su } \\
\text { disponibilidad de tiempo. }\end{array}$ & 1 & 2 & 3 & 4 & 5 & 6 & 7 & 8 & 9 & 10 & $\mathrm{NR} / \mathrm{NS}$ & \\
\hline $\begin{array}{l}\text { 11. Corrigen de forma inmediata cualquier error en } \\
\text { el servicio. }\end{array}$ & 1 & 2 & 3 & 4 & 5 & 6 & 7 & 8 & 9 & 10 & NR/NS & \\
\hline 12. Es consistente en el servicio que brinda. & 1 & 2 & 3 & 4 & 5 & 6 & 7 & 8 & 9 & 10 & NR/NS & \\
\hline 13. El cobro se realiza en forma rápida y sin errores. & 1 & 2 & 3 & 4 & 5 & 6 & 7 & 8 & 9 & 10 & NR/NS & \\
\hline 14. Sirven la comida en la forma en que se pidió. & 1 & 2 & 3 & 4 & 5 & 6 & 7 & 8 & 9 & 10 & NR/NS & \\
\hline $\begin{array}{l}\text { 15. Durante los horarios más concurridos los } \\
\text { empleados se ayudan unos a otros para mantener la } \\
\text { velocidad y la calidad del servicio. }\end{array}$ & 1 & 2 & 3 & 4 & 5 & 6 & 7 & 8 & 9 & 10 & NR/NS & \\
\hline 16. Proporciona un servicio oportuno y rápido. & 1 & 2 & 3 & 4 & 5 & 6 & 7 & 8 & 9 & 10 & NR/NS & \\
\hline $\begin{array}{l}\text { 17. Los empleados realizan un esfuerzo extra para } \\
\text { servir los pedidos especiales. }\end{array}$ & 1 & 2 & 3 & 4 & 5 & 6 & 7 & 8 & 9 & 10 & NR/NS & \\
\hline $\begin{array}{l}\text { 18. Tiene empleados que pueden responder a sus } \\
\text { preguntas de forma eficiente. }\end{array}$ & 1 & 2 & 3 & 4 & 5 & 6 & 7 & 8 & 9 & 10 & NR/NS & \\
\hline 19. El trato del personal lo hace sentir bien y cómodo. & 1 & 2 & 3 & 4 & 5 & 6 & 7 & 8 & 9 & 10 & NR/NS & \\
\hline $\begin{array}{l}\text { 20. Los empleados tienen el conocimiento para } \\
\text { contestar todas las preguntas sobre el platillo que } \\
\text { sirven en materia de ingredientes y forma de } \\
\text { prepararlos. }\end{array}$ & 1 & 2 & 3 & 4 & 5 & 6 & 7 & 8 & 9 & 10 & NR/NS & \\
\hline $\begin{array}{l}\text { 21. Los empleados los hicieron sentirse seguros en } \\
\text { todo momento. }\end{array}$ & 1 & 2 & 3 & 4 & 5 & 6 & 7 & 8 & 9 & 10 & NR/NS & \\
\hline $\begin{array}{l}\text { 22. Tiene personal capacitado, competente y } \\
\text { experimentado. }\end{array}$ & 1 & 2 & 3 & 4 & 5 & 6 & 7 & 8 & 9 & 10 & NR/NS & \\
\hline $\begin{array}{l}\text { 23. Sintió que la administración apoya al personal } \\
\text { para que haga bien su trabajo }\end{array}$ & 1 & 2 & 3 & 4 & 5 & 6 & 7 & 8 & 9 & 10 & NR/NS & \\
\hline $\begin{array}{l}\text { 24. El personal es sensible a sus necesidades en lugar } \\
\text { de acatar las políticas y reglas del servicio. }\end{array}$ & 1 & 2 & 3 & 4 & 5 & 6 & 7 & 8 & 9 & 10 & NR/NS & \\
\hline 25. Le hicieron sentir como alguien especial. & 1 & 2 & 3 & 4 & 5 & 6 & 7 & 8 & 9 & 10 & NR/NS & \\
\hline 26. Los empleados anticiparon sus necesidades & 1 & 2 & 3 & 4 & 5 & 6 & 7 & 8 & 9 & 10 & NR/NS & \\
\hline $\begin{array}{l}\text { 27. Los empleados son receptivos y ven al cliente con } \\
\text { simpatía si algo sale mal. }\end{array}$ & 1 & 2 & 3 & 4 & 5 & 6 & 7 & 8 & 9 & 10 & $\mathrm{NR} / \mathrm{NS}$ & \\
\hline $\begin{array}{l}\text { 28. Los empleados parecen tener siempre en mente } \\
\text { los interés del cliente }\end{array}$ & 1 & 2 & 3 & 4 & 5 & 6 & 7 & 8 & 9 & 10 & NR/NS & \\
\hline
\end{tabular}


Anexo 2. Puntaje promedio asignado a cada dimensión por atributo según perfil del usuario. Universidad de Costa Rica, 2016

\begin{tabular}{|c|c|c|c|c|c|c|c|c|c|c|c|c|c|c|c|c|c|}
\hline$\tilde{a}$ & तิ & $\stackrel{\circ}{i}$ & ণ્ণิ & $\stackrel{\circ}{\vec{i}}$ & $\stackrel{q}{-}$ & $\stackrel{8}{:}$ & $\stackrel{R}{i}$ & $\stackrel{9}{\leftrightarrows}$ & $\stackrel{8}{-}$ & $\begin{array}{l}\infty \\
\infty \\
0\end{array}$ & $\mid$ & $\stackrel{\varrho}{\leftrightarrows}$ & $\stackrel{8}{-}$ & $\stackrel{8}{8}$ & R̊ & $\stackrel{\circ}{\leftrightarrows}$ & $\begin{array}{l}\hat{\imath} \\
\hat{0}\end{array}$ \\
\hline 音 & 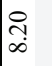 & $\begin{array}{l}8 \\
\infty \\
\infty\end{array}$ & స్ & $\begin{array}{l}: \\
\text { in } \\
\infty\end{array}$ & $\stackrel{q}{q}$ & $\begin{array}{l}\stackrel{2}{\infty} \\
\infty\end{array}$ & $\underset{\sim}{\stackrel{8}{0}}$ & ల్ & $\stackrel{\circ}{a}$ & in & $\begin{array}{l}\vec{b} \\
\infty \\
\infty\end{array}$ & సે & $\begin{array}{l}q \\
a\end{array}$ & in & $\stackrel{R}{\stackrel{2}{a}}$ & $\stackrel{q}{q}$ & ڤેे \\
\hline$\tilde{a}$ & $\stackrel{9}{-1}$ & $\stackrel{9}{9}$ & $\stackrel{?}{i}$ & $\stackrel{\circ}{\stackrel{i}{i}}$ & तิ & ণ্ల్) & $\underset{i}{\stackrel{B}{i}}$ & $\stackrel{\circ}{i}$ & ત્તి & $\underset{\text { î }}{\text { in }}$ & $\stackrel{\infty}{\stackrel{2}{\longrightarrow}}$ & $\stackrel{?}{\rightarrow}$ & $\stackrel{8}{-}$ & $\underset{i}{8}$ & $\stackrel{n}{\rightarrow}$ & i্ & . \\
\hline 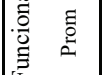 & $\underset{\infty}{\infty}$ & $\begin{array}{l}8 \\
\infty \\
\infty\end{array}$ & $\stackrel{\circ}{a}$ & 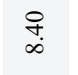 & $\underset{\infty}{\infty}$ & ત్రి & $\stackrel{n}{n}$ & $\underset{\infty}{\infty}$ & $\underset{\infty}{\stackrel{\Upsilon}{~}}$ & $\underset{\infty}{8}$ & $\vec{\infty}$ & $\underset{\infty}{\stackrel{R}{R}}$ & $\begin{array}{l}8 \\
\infty \\
\infty\end{array}$ & $\underset{\infty}{\stackrel{R}{0}}$ & $\stackrel{\circ}{a}$ & 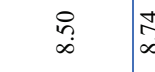 & $\infty$ \\
\hline$\tilde{a}$ & în. & $\stackrel{\circ}{i}$ & $\stackrel{?}{+}$ & $\stackrel{9}{9}$ & $\stackrel{q}{i}$ & $\underset{\text { i }}{\text { i }}$ & $\begin{array}{l}\text { in } \\
\text { in }\end{array}$ & $\stackrel{?}{\stackrel{f}{-}}$ & $\stackrel{\infty}{\stackrel{\infty}{-}}$ & $\underset{\text { i }}{\stackrel{\text { in }}{ }}$ & 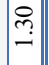 & $\stackrel{9}{-}$ & $\stackrel{8}{:}$ & $\stackrel{\circ}{i}$ & . & $\underset{i}{\stackrel{i}{i}}$ & I: \\
\hline हี & $\stackrel{?}{i}$ & 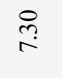 & $\underset{\infty}{\infty}$ & $\stackrel{0}{\infty}$ & $\stackrel{\leftrightarrow}{\leftrightarrow}$ & $\stackrel{9}{ }$ & $\underset{0}{8}$ & $\begin{array}{l}n \\
\text { in } \\
\infty\end{array}$ & $\underset{\infty}{\stackrel{0}{0}}$ & $\underset{\infty}{q}$ & $\underset{\substack{+\infty \\
r}}{r}$ & 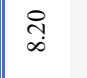 & $\begin{array}{l}n \\
\infty \\
\infty \\
\infty\end{array}$ & $\underset{\infty}{\stackrel{q}{+}}$ & 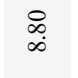 & $\stackrel{0}{\infty}$ & 0 \\
\hline$\tilde{a}$ & 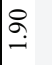 & $\stackrel{9}{9}$ & $\underset{-}{\stackrel{\leftrightarrow}{-}}$ & $\stackrel{\circ}{\stackrel{i}{i}}$ & $\stackrel{q}{g}$ & $\stackrel{\text { q }}{-}$ & $\underset{i}{\stackrel{q}{i}}$ & $\stackrel{\oplus}{-}$ & $\stackrel{\text { q }}{-}$ & $\stackrel{\infty}{\stackrel{\oplus}{-}}$ & $\vec{ָ}$ & $\stackrel{0}{=}$ & $\stackrel{\circ}{\leftrightarrows}$ & $\stackrel{n}{n}$ & 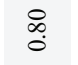 & $\stackrel{?}{i}$ & $\stackrel{\text { oे }}{\text {. }}$ \\
\hline 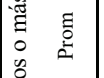 & $\underset{\substack{\infty \\
\infty \\
\infty}}{.}$ & 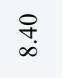 & તે & $\underset{\infty}{\infty}$ & $\stackrel{8}{\circ}$ & $\begin{array}{l}\infty \\
\infty \\
\infty\end{array}$ & $\underset{\infty}{\stackrel{\infty}{0}}$ & $\begin{array}{l}\stackrel{2}{\infty} \\
\infty\end{array}$ & $\begin{array}{l}\infty \\
\infty \\
\infty\end{array}$ & $\begin{array}{l}8 \\
\infty \\
\infty\end{array}$ & $\begin{array}{l}\widetilde{T} \\
\infty \\
\infty \\
\infty\end{array}$ & $\stackrel{\circ}{\circ}$ & $\stackrel{\circ}{\circ}$ & $\stackrel{\circ}{\circ}$ & in & $\begin{array}{l}\infty \\
\infty \\
\infty\end{array}$ & oे. \\
\hline$\tilde{a}$ & तิ & $\stackrel{?}{i}$ & $\stackrel{\circ}{\rightarrow}$ & $\underset{i}{\stackrel{P}{i}}$ & 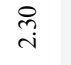 & $\stackrel{\text { i }}{\circ}$ & $\begin{array}{l}\stackrel{0}{n} \\
\text { in }\end{array}$ & 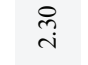 & $\underset{\substack{i \\
i}}{ }$ & : & $\underset{\sim}{\vec{i}}$ & $\stackrel{\infty}{\stackrel{\infty}{-}}$ & $\stackrel{n}{n}$ & $\stackrel{?}{i}$ & $\stackrel{\infty}{\rightarrow}$ & 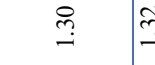 & ڤึ? \\
\hline 总 & $\stackrel{?}{i}$ & $\stackrel{n}{r}$ & $\begin{array}{l}\infty \\
\infty \\
\infty \\
\infty\end{array}$ & $\stackrel{?}{i}$ & 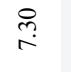 & $\stackrel{?}{6}$ & $\stackrel{\leftrightarrow}{\circ}$ & $\underset{\infty}{\stackrel{\infty}{\infty}}$ & $\stackrel{i n}{r}$ & $\begin{array}{l}\infty \\
\infty \\
\infty\end{array}$ & 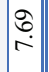 & $\begin{array}{l}0 \\
\infty \\
\infty\end{array}$ & $\underset{\infty}{\stackrel{P}{\infty}}$ & $\underset{\infty}{\stackrel{P}{\infty}}$ & $\underset{\infty}{\stackrel{\circ}{\infty}}$ & $\underset{\infty}{\stackrel{0}{0}}$ & 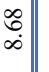 \\
\hline$\tilde{a}$ & in & 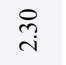 & $\stackrel{8}{\stackrel{B}{-}}$ & $\stackrel{\circ}{\stackrel{i}{i}}$ & $\underset{i}{i}$ & $\stackrel{\stackrel{+}{i}}{i}$ & $\stackrel{i}{i}$ & $\stackrel{8}{:}$ & $\underset{i}{\stackrel{i}{i}}$ & ત્సి & : & $\underset{i}{\stackrel{i}{i}}$ & $\stackrel{?}{?}$ & त् & : & $\stackrel{\circ}{i}$ & \\
\hline 咅 & $\stackrel{?}{i}$ & ণิ & $\underset{\infty}{\stackrel{R}{\infty}}$ & 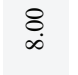 & $\stackrel{?}{i}$ & 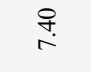 & ه. & $\underset{\infty}{\stackrel{q}{q}}$ & $\stackrel{\infty}{\stackrel{\infty}{\sim}}$ & $\stackrel{\circ}{\infty}$ & : & $\underset{\infty}{\infty}$ & $\begin{array}{l}0 \\
\infty \\
\infty\end{array}$ & $\underset{\infty}{\infty}$ & $\begin{array}{l}\infty \\
\infty \\
\infty\end{array}$ & $\stackrel{8}{9}$ & \\
\hline n & 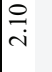 & $\underset{i}{\stackrel{8}{0}}$ & 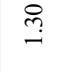 & $\stackrel{8}{\circ}$ & 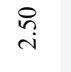 & $\underset{i}{\stackrel{q}{i}}$ & $\underset{i}{\stackrel{q}{i}}$ & $\stackrel{\overbrace{}}{-}$ & $\stackrel{8}{-}$ & $\underset{i}{\stackrel{8}{i}}$ & $\stackrel{n}{=}$ & $\stackrel{9}{9}$ & $\stackrel{8}{-}$ & $\stackrel{\circ}{i}$ & $\stackrel{\leftrightarrow}{-}$ & $\underset{i}{\stackrel{\leftrightarrow}{i}}$ & \& \\
\hline 音 & 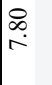 & $\stackrel{n}{n}$ & $\stackrel{8}{\circ}$ & ల్లి & $\stackrel{8}{\stackrel{\leftrightarrow}{n}}$ & $\stackrel{8}{\stackrel{1}{r}}$ & : & $\underset{\infty}{\stackrel{R}{\infty}}$ & $\begin{array}{l}\text { Î } \\
\infty\end{array}$ & $\begin{array}{l}0 \\
\infty \\
\infty \\
\infty\end{array}$ & 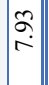 & $\stackrel{\circ}{\stackrel{\infty}{\infty}}$ & $\underset{\infty}{\stackrel{0}{0}}$ & $\begin{array}{l}0 \\
\infty \\
\infty\end{array}$ & $\begin{array}{l}\infty \\
\infty \\
\infty\end{array}$ & స్ & $\vec{n}$ \\
\hline 5 & $=$ & $\sim$ & ' & 은 & - & $a$ & ' & , & - & $\sigma$ & & 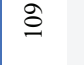 & $\bar{\lambda}$ & in & r & $\mathscr{F}$ & \\
\hline$\tilde{a}$ & în & $\stackrel{\circ}{i}$ & $\stackrel{\text { I. }}{-}$ & $\stackrel{8}{9}$ & $\underset{i}{i}$ & $\stackrel{\stackrel{+}{i}}{\mathrm{i}}$ & in & $\stackrel{q}{\rightarrow}$ & $\stackrel{\infty}{\infty}$ & $\underset{i}{\stackrel{\leftrightarrow}{i}}$ & 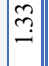 & $\stackrel{8}{9}$ & $\stackrel{8}{\stackrel{8}{\circ}}$ & $\stackrel{\circ}{i}$ & $\stackrel{8}{\circ}$ & $\underset{i}{\stackrel{8}{i}}$ & 守 \\
\hline ह & 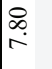 & 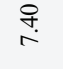 & $\begin{array}{l}8 \\
\infty \\
\infty\end{array}$ & $\begin{array}{l}\text { तి } \\
\infty\end{array}$ & $\stackrel{\infty}{\sim}$ & 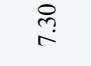 & $\underset{0}{\otimes}$ & $\begin{array}{c}0 \\
\infty \\
\infty\end{array}$ & $\stackrel{?}{\infty}$ & $\underset{\infty}{\substack{q \\
\infty}}$ & $\frac{\sigma}{r}$ & $\underset{\infty}{\infty}$ & $\begin{array}{l}\stackrel{8}{0} \\
\infty\end{array}$ & $\begin{array}{l}\stackrel{\wp}{n} \\
\infty\end{array}$ & $\begin{array}{l}\stackrel{8}{8} \\
\infty\end{array}$ & ণి & 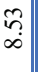 \\
\hline$=$ & $\stackrel{\infty}{\infty}$ & 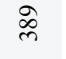 & $\overline{\mathrm{m}}$ & $\overrightarrow{\Phi_{m}}$ & \& & $\underset{\widetilde{\infty}}{\tilde{m}}$ & $\overline{\mathrm{n}}$ & $\overline{\tilde{m}}$ & मे & 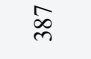 & $\overrightarrow{\tilde{m}}$ & $\underset{\widetilde{\sim}}{\sim}$ & $\stackrel{2}{m}$ & 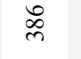 & $\underset{m}{ \pm}$ & $\stackrel{\infty}{m}$ & $\bar{m}$ \\
\hline 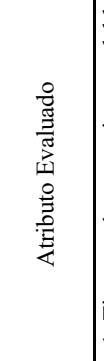 & 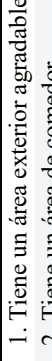 & 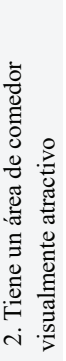 & 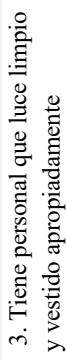 & 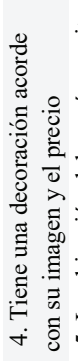 & 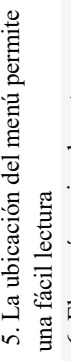 & 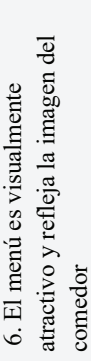 & 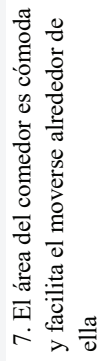 & 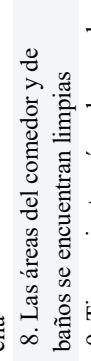 & 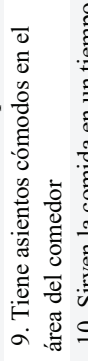 & 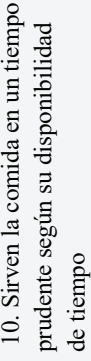 & 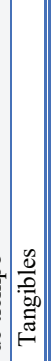 & 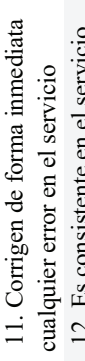 & 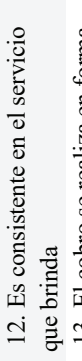 & 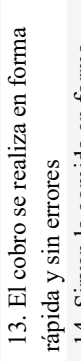 & 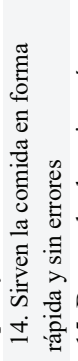 & 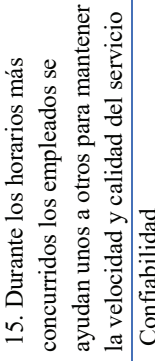 & 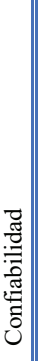 \\
\hline
\end{tabular}




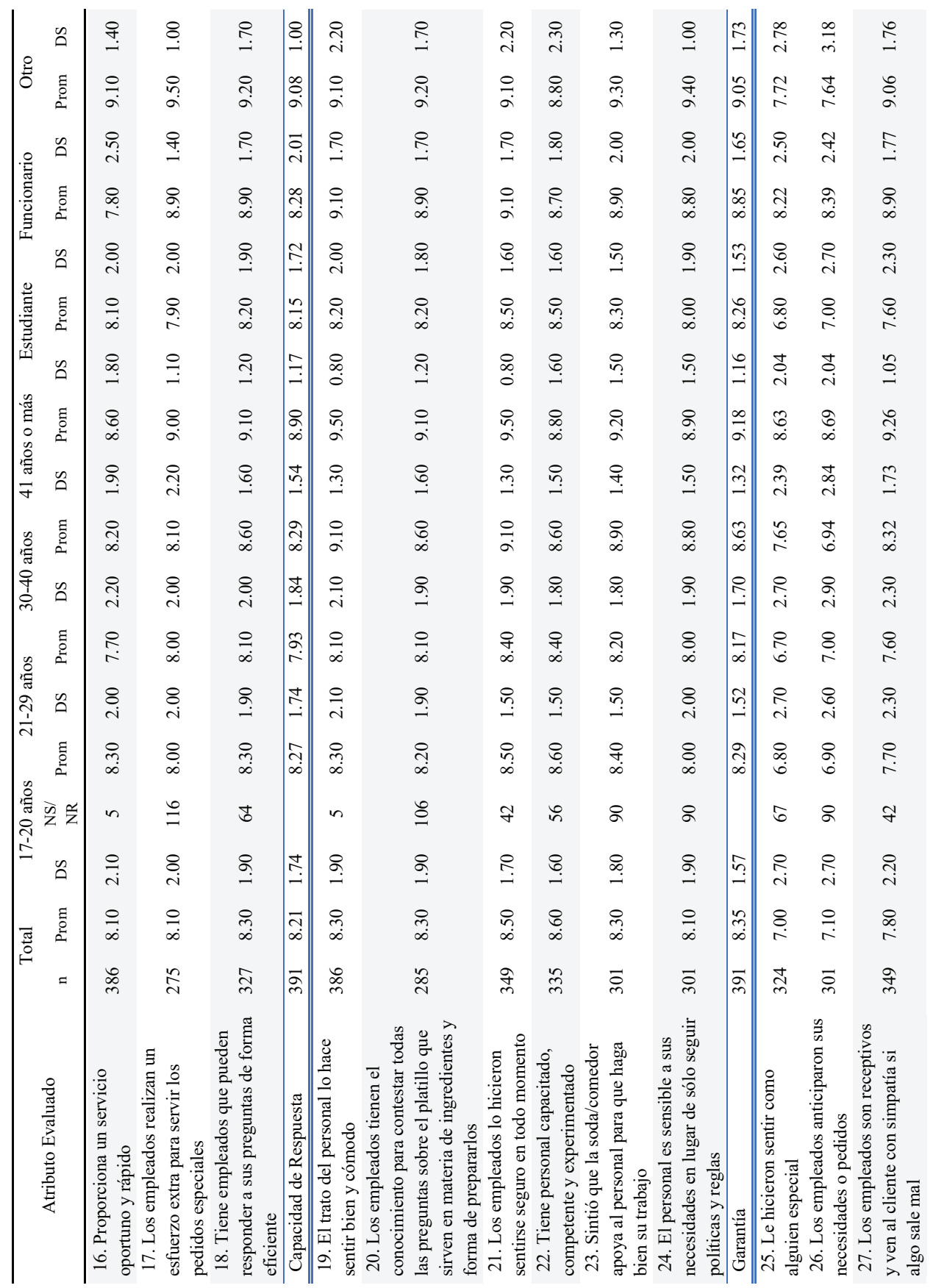




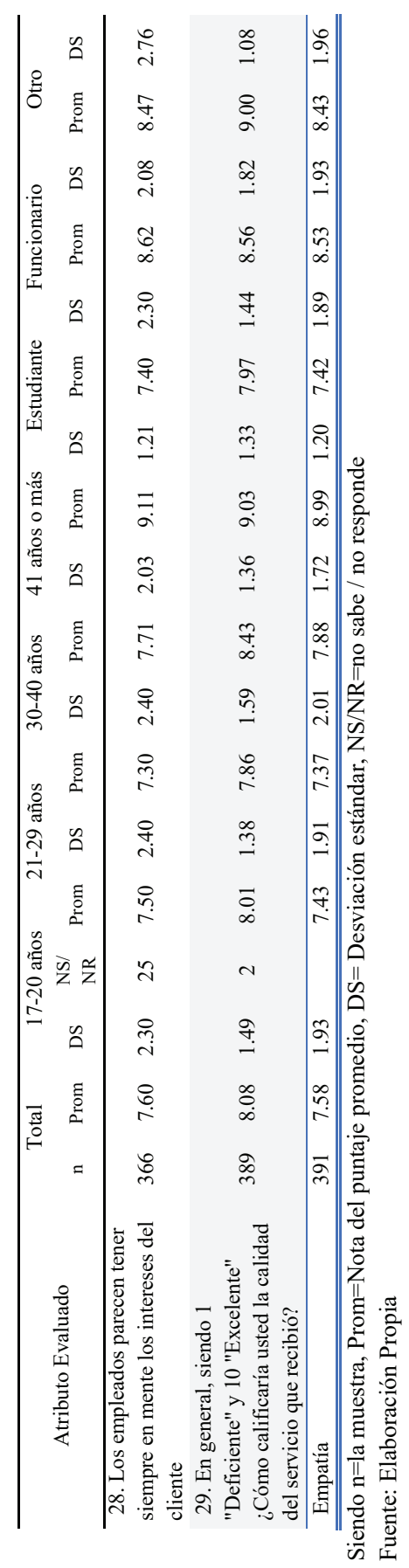

